\title{
Complex shock structure in the western hot-spot of Pictor A
}

\author{
C. J. Saxton ${ }^{1,2}$, R. S. Sutherland ${ }^{2}$, G. V. Bicknell ${ }^{1,2}$, G. F. Blanchet ${ }^{1,2,3}$, and S. J. Wagner ${ }^{4}$ \\ 1 Department of Physics \& Theoretical Physics, Australian National University, Canberra, Australia \\ 2 Research School of Astronomy \& Astrophysics, Australian National University, Canberra, Australia \\ e-mail: saxton@mso.anu.edu.au, ralph@mso.anu.edu.au \\ 3 INSA, 135 avenue de Rangueil, 31077 Toulouse Cedex 4, France \\ ${ }^{4}$ Landessternwarte Heidelberg-Konigstuhl, Konigstuhl, 69117 Heidelberg, Germany \\ e-mail: swagner@lsw.uni-heidelberg.de
}

Received 16 March 2001 / Accepted 28 June 2002

\begin{abstract}
We have carried out simulations of supersonic light jets in order to model the features observed in optical and radio images of the western hot-spot in the radio galaxy Pictor A. We have considered jets with density ratios $\eta=10^{-2}-10^{-4}$, and Mach numbers ranging between 5 and 50. From each simulation, we have generated ray-traced maps of radio surface brightness at a variety of jet inclinations, in order to study the appearance of time-dependent luminous structures in the vicinity of the western hot-spot. We compare these rendered images with observed features of Pictor A. A remarkable feature of Pictor A observations is a bar-shaped "filament" inclined almost at right angles to the inferred jet direction and extending 24" $\left(10.8 \mathrm{~h}^{-1} \mathrm{kpc}\right)$ along its longest axis. The constraints of reproducing the appearance of this structure in simulations indicate that the jet of Pictor A lies nearly in the plane of the sky. The results of the simulation are also consistent with other features found in the radio image of Pictor A. This filament arises from the surging behaviour of the jet near the hot-spot; the surging is provoked by alternate compression and decompression of the jet by the turbulent backflow in the cocoon. We also examine the arguments for the jet in Pictor A being at a more acute angle to the line of sight and find that our preferred orientation is just consistent with the limits on the brightness ratio of the X-ray jet and counter-jet. We determine from our simulations, the structure function of hot-spot brightness and also the cumulative distribution of the ratio of intrinsic hot-spot brightnesses. The latter may be used to quantify the use of hot-spot ratios for the estimation of relativistic effects.
\end{abstract}

Key words. galaxies: jets - galaxies: general

\section{Introduction}

The FR II radio galaxy Pictor A $(z=0.0342)$ consists of two lobes dominated by hot-spots separated by $4^{\prime}\left(210 h^{-1} \mathrm{kpc}\right)$ from the flat spectrum nucleus. Near the western hot-spot, there is a faint, $24^{\prime \prime}\left(10.8 h^{-1} \mathrm{kpc}\right)$ long radio and optical filament, "upstream of the hot-spot" first detected by Röser \& Meisenheimer (1987). Wagner et al. (2002) found the filament to be highly polarised, confirming that it radiates via optical synchrotron emission.

The filament has been studied in detail at several radio frequencies by Perley et al. (1997). The radio emission at $8415 \mathrm{MHz}$, Fig. 1, which bears a striking correspondence to the optical emission, also reveals a knot further upstream from the filament. This is of particular interest since at various timesteps throughout our simulation such a morphology is captured.

The discovery of optical synchrotron radiation, emitted by electrons with radiative life-times of about $100 \mathrm{yr}$ (for magnetic fields of the order of equipartition), in a filament which extends

Send offprint requests to: G. V. Bicknell, e-mail: Geoff.Bicknell@mso.anu.edu.au several thousand light years perpendicular to the jet strongly indicates that the electrons are accelerated in situ. The crossing time from the hot-spot to the filament is much longer than the cooling time-scale, and efficient acceleration to optical emitting energies is required.

Our objective here is to ascertain whether purely hydrodynamical simulations can qualitatively reproduce the radio and optical morphology. Indeed on the basis of our simulations we suggest below that the filament is the result of a quasi-periodic pulsing behaviour of the terminal jet shock.

\section{Summary of the observations}

Before discussing the simulations, we summarise relevant aspects of the observations and in the following sections we point out the relevance of the features in the simulations to the observational data.

A total intensity radio map (Perley et al. 1997), displayed in Fig. 1 shows the high-surface brightness western hot-spot; a bar-shaped region (the filament) east of the hot-spot, extends perpendicular to the inferred direction of the jet. The region 


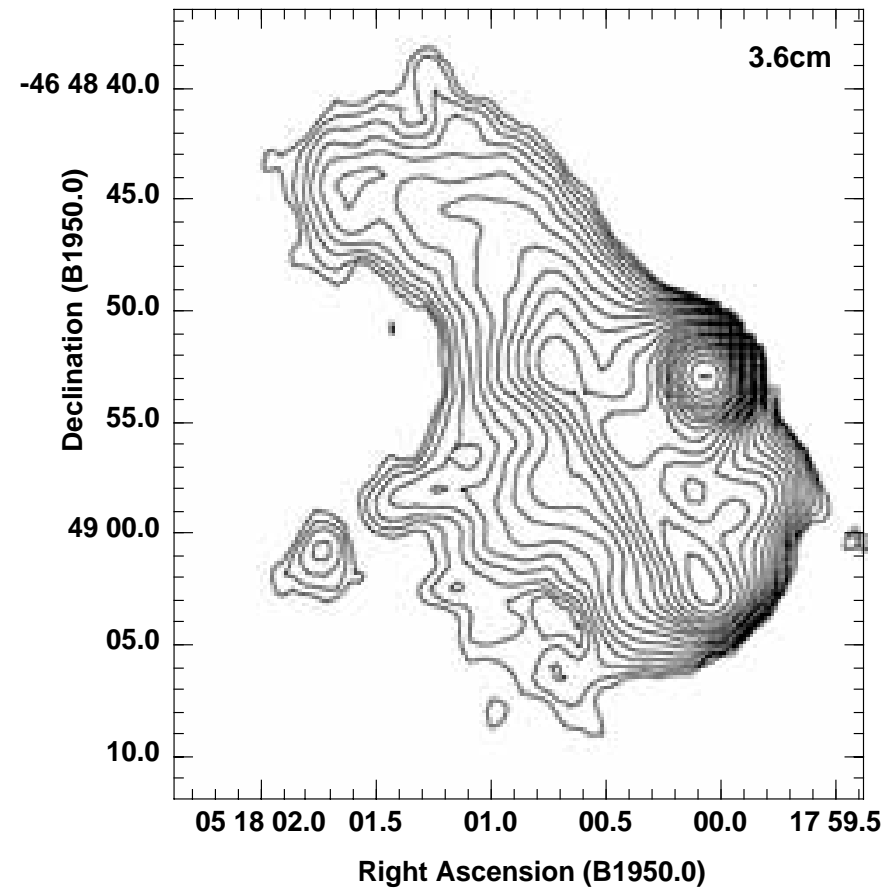

Fig. 1. Radio image. VLA $8415 \mathrm{MHz}$ map of the western lobe, with contour levels at multiples of $1 / \sqrt{2}$ (Perley et al. 1997).

between the hot-spot and the ridge line of the bar shows a "pedestal" of lower surface brightness.

High S/N observations were obtained with the VLT (UT1) during commissioning of FORS1. Figure 2 shows total intensity emission at $450 \mathrm{~nm}$. Wagner et al. (2002) presented polarization maps derived from these data, showing up to $60 \%$ polarization in the hot-spot, and up to $40 \%$ polarization in the filament. Such a high degree of polarization cannot be the result of scattering and strongly suggests that the filament radiates optical synchrotron emission. Moreover, in the optical image, the bar-shaped filament is quite bright, and spatially coincides with a similar in the radio images. The eastern edge of the bar is very pronounced, but the surface brightness gradient in the western direction is more gradual. The filament is brightest at its ends and near the centre, where it overlaps the inferred jet direction. In the optical image there are faint extensions from the hot-spot extending in the direction of the ends of the filament.

\section{Simulation methodology}

\subsection{Hydrodynamics}

The earliest simulations of astrophysical jets were published by Norman et al. (1982) and Smith et al. (1985). Their simulations revealed hot-spot morphologies that were different from the simple one-shock structure envisaged by Blandford \& Rees (1974). These papers were seminal in leading to a good physical understanding of the properties of supersonic extragalactic jets.

In this work we have carried out similar simulations with a view to addressing in detail the time variability of hot-spot morphology. In particular we address the question of whether the Pictor A morphology could arise naturally in a light

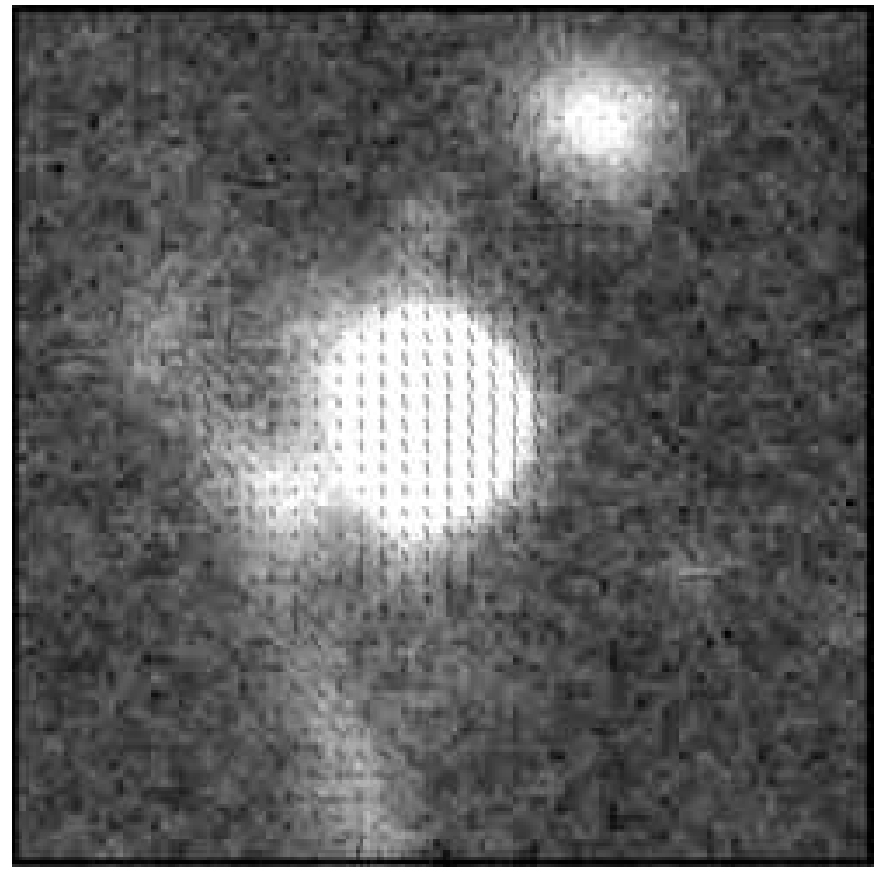

Fig. 2. Optical image. Total intensity emission at $450 \mathrm{~nm}$ with polarization map overlay.

supersonic jet. Our simulations were conducted using the VH-1 code (Blondin \& Lukfin 1993) which is an implementation of the Piecewise Parabolic Method (PPM) (Colella \& Woodward 1984). An advantage of PPM for this type of simulation is its excellent resolution of shocks.

The simulations were computed on an axisymmetric grid, with $600 \times 300$ cells in the axial and radial directions. We performed calculations with nine different cases of the jet parameters, i.e. jet Mach number $M=5,10,50$ and the ratio of jet to background densities, $\eta=10^{-2}, 10^{-3}, 10^{-4}$. In each case the jet density and pressure were normalized by the respective values, $\left(\rho_{0}, p_{0}\right)$, in the background medium. If we take $T=10^{7} T_{7} \mathrm{~K}$ to be the temperature of the external medium, then the normalising value of the velocity is $v_{0}=\sqrt{p_{0} / \rho_{0}}=\sqrt{k T / \mu m_{\mathrm{p}}} \approx 3.65 \times 10^{7} T_{7}^{1 / 2} \mathrm{~cm} \mathrm{~s}^{-1}$. The unit of length, $x_{0}$, is defined by the value of the jet radius $=0.25$, in numerical units. If we take the observed western hot-spot $F W H M \approx 1.2^{\prime \prime} \approx 2.7 \times 10^{21} \mathrm{~cm}$, for $H_{0}=70 \mathrm{~km} \mathrm{~s}^{-1} \mathrm{Mpc}^{-1}$, as indicative of the jet diameter, then $x_{0} \approx 9.0 \times 10^{21} \mathrm{~cm}$ and the unit of time, $t_{0}=x_{0} v_{0}^{-1} \approx 9.4 \times 10^{6} T_{7}^{-1 / 2} \mathrm{yr}$. As with simulations of this type, the exact physical parameters of velocity and density are not recovered. However, a high Mach number and low density ratio serve to reproduce, at least qualitatively, many of the features found in real jets.

Table 1 lists the jet parameters, time resolution and left boundary condition of our simulations. We define the grid to be 10 units in the $z$ direction and 5 units in the radial direction. Table 2 indicates the jet's total mass flux, force and power for each choice of the basic parameters, in terms of fiducial values of the temperature, $T_{7}$, and the number density of the background medium, $n_{-3}=n / 10^{-3} \mathrm{~cm}^{-3}$. For a jet with Mach number $M$, density contrast $\eta$, adiabatic index $\gamma$ (assumed equal 
Table 1. Simulation physical parameters $(\eta, M)$, time coverage $\left(t_{\text {init }} \leq t \leq t_{\text {end }}\right)$ and frame intervals $(\Delta t)$. Times are expressed in terms of the scale $t_{0}$.

\begin{tabular}{lcrlllc}
\hline \hline label & left boundary & $\eta$ & $\mathrm{M}$ & $t_{\text {init }}$ & $t_{\text {end }}$ & $\Delta t$ \\
\hline C2V & closed & $10^{-2}$ & 5 & 0 & 2.4 & $4 \times 10^{-3}$ \\
C3V & closed & $10^{-3}$ & 5 & 0 & 2.4 & $4 \times 10^{-3}$ \\
C4V & closed & $10^{-4}$ & 5 & 0 & 2.4 & $4 \times 10^{-3}$ \\
C2X & closed & $10^{-2}$ & 10 & 0 & 1.2 & $2 \times 10^{-3}$ \\
C3X & closed & $10^{-3}$ & 10 & 0 & 1.2 & $2 \times 10^{-3}$ \\
C4X & closed & $10^{-4}$ & 10 & 0 & 1.2 & $2 \times 10^{-3}$ \\
C2L & closed & $10^{-2}$ & 50 & 0 & 0.24 & $4 \times 10^{-4}$ \\
C3L & closed & $10^{-3}$ & 50 & 0 & 0.24 & $4 \times 10^{-4}$ \\
C4L & closed & $10^{-4}$ & 50 & 0 & 0.24 & $4 \times 10^{-4}$ \\
& & & & & & \\
c3V & closed & $10^{-3}$ & 5 & 1.6 & 2.4 & $4 \times 10^{-4}$ \\
C4V & closed & $10^{-4}$ & 5 & 0.8 & 1.28 & $2 \times 10^{-4}$ \\
C3X & closed & $10^{-3}$ & 10 & 0.8 & 1.2 & $2 \times 10^{-4}$ \\
C4X & closed & $10^{-4}$ & 10 & 0.4 & 0.64 & $1 \times 10^{-4}$ \\
C3L & closed & $10^{-3}$ & 50 & 0.05 & 0.2 & $5 \times 10^{-5}$ \\
& & & & & & \\
\hline
\end{tabular}

to the ambient medium), and cross-sectional area $A=\pi r_{j}^{2}$, the initial flow velocity, mass flux, force and kinetic power are

$v_{\mathrm{j}}=v_{0} \sqrt{\frac{\gamma}{\eta}} M$

$\dot{M}_{\mathrm{j}}=A \rho_{0} v_{0} \sqrt{\gamma \eta} M$

$F_{\mathrm{j}}=A\left(\rho v^{2}+p\right)=A \rho_{0} v_{0}^{2}\left(\gamma M^{2}+1\right)$, and

$P_{\mathrm{j}}=A\left(\frac{1}{2} \rho v^{3}+\frac{\gamma}{\gamma-1} p v\right)=A \rho_{0} v_{0}^{3}\left(\frac{1}{2} M^{2}+\frac{1}{\gamma-1}\right) M \sqrt{\frac{\gamma^{3}}{\eta}}$

These are subrelativistic expressions, although the jets of Pictor A may involve relativistic flows of plasma. One may relate the $(\eta, M)$ parameters of a classical jet to an equivalent pair of parameters for a relativistic jet with equivalent thrust or power, e.g. Rosen et al. (1999), Carvalho \& O'Dea (2002). However we are not modelling the system to determine the Lorentz factor of the Pictor A jet to great precision.

Each simulation is initialised with the high-velocity, lowdensity jet material extruding a small distance into the ambient medium. At every subsequent time step the conditions of the initial jet are recreated in the cells at the base of the jet on the left (inner $z$ ) boundary of the grid, and thus the jet's injected fluxes of mass, momentum and energy remain constant in time. We performed simulations with two different choices for the condition applied at the left boundary outside the radius of the jet. (1) A reflecting ("closed") boundary condition represents the effects of the symmetry plane through the nucleus, felt by a jet that is close to its origin and counterjet. (2) An "open" boundary allows free outflow of mass, momentum and energy, thereby representing systems where the hot-spot and bow shock are distant from the nucleus and not directly influenced by the opposite lobe. The outer $z$ and $r$ boundaries are open to outflow.

\begin{tabular}{lcrlllc}
\hline \hline label & left boundary & $\eta$ & $\mathrm{M}$ & $t_{\text {init }}$ & $t_{\text {end }}$ & $\Delta t$ \\
\hline 02V & open & 5 & $10^{-2}$ & 0 & 2.4 & $4 \times 10^{-3}$ \\
03V & open & 5 & $10^{-3}$ & 0 & 2.4 & $4 \times 10^{-3}$ \\
04V & open & 5 & $10^{-4}$ & 0 & 2.4 & $4 \times 10^{-3}$ \\
02X & open & 10 & $10^{-2}$ & 0 & 1.2 & $2 \times 10^{-3}$ \\
03X & open & 10 & $10^{-3}$ & 0 & 1.2 & $2 \times 10^{-3}$ \\
04X & open & 10 & $10^{-4}$ & 0 & 1.2 & $2 \times 10^{-3}$ \\
02L & open & 50 & $10^{-2}$ & 0 & 0.24 & $4 \times 10^{-4}$ \\
03L & open & 50 & $10^{-3}$ & 0 & 0.24 & $4 \times 10^{-4}$ \\
04L & open & 50 & $10^{-4}$ & 0 & 0.24 & $4 \times 10^{-4}$ \\
& & & & & & \\
03V & open & 5 & $10^{-3}$ & 1.0 & 2.4 & $4 \times 10^{-4}$ \\
04V & open & 5 & $10^{-4}$ & 1.6 & 2.4 & $2 \times 10^{-4}$ \\
03X & open & 10 & $10^{-3}$ & 0.2 & 1.0 & $2 \times 10^{-4}$ \\
04X & open & 10 & $10^{-4}$ & 0.8 & 1.2 & $1 \times 10^{-4}$ \\
03L & open & 50 & $10^{-3}$ & 0.03 & 0.15 & $5 \times 10^{-5}$ \\
04L & open & 50 & $10^{-4}$ & 0.02 & 0.18 & $5 \times 10^{-5}$ \\
\hline
\end{tabular}

Table 2. Velocity, mass flux, force and power of a jet with radius $r_{\mathrm{j}}=0.25 x_{0}$ for our choices of the parameters $(\eta, M)$. These are nonrelativistic evaluations, assuming a number density of $10^{-2} \mathrm{~cm}^{-2}$ and a temperature of $10^{7} \mathrm{~K}$ in the ambient medium.

\begin{tabular}{rccclc}
\hline \hline$M$ & $\eta$ & $\begin{array}{c}\frac{v_{\mathrm{j}}}{T_{7}^{1 / 2}} \\
\left(\mathrm{~cm} \mathrm{~s}^{-1}\right)\end{array}$ & $\begin{array}{c}\frac{\dot{M}_{\mathrm{j}}}{n_{-3} T_{7}^{1 / 2}} \\
\left(M_{\odot} \mathrm{yr}^{-1}\right)\end{array}$ & $\begin{array}{l}\frac{F_{\mathrm{j}}}{n_{-3} T_{7}} \\
(\mathrm{dyn})\end{array}$ & $\begin{array}{c}\frac{P_{\mathrm{j}}}{n_{-3} T_{7}^{3 / 2}} \\
\left(\mathrm{erg} \mathrm{s}^{-1}\right)\end{array}$ \\
\hline 5 & $10^{-2}$ & $2.36 \times 10^{9}$ & $1.39 \times 10^{-2}$ & $2.11 \times 10^{33}$ & $2.72 \times 10^{42}$ \\
5 & $10^{-3}$ & $7.45 \times 10^{9}$ & $4.40 \times 10^{-3}$ & $2.11 \times 10^{33}$ & $8.61 \times 10^{42}$ \\
5 & $10^{-4}$ & $2.36 \times 10^{10}$ & $1.39 \times 10^{-3}$ & $2.11 \times 10^{33}$ & $2.72 \times 10^{43}$ \\
10 & $10^{-2}$ & $4.71 \times 10^{9}$ & $2.78 \times 10^{-2}$ & $8.31 \times 10^{33}$ & $2.00 \times 10^{43}$ \\
10 & $10^{-3}$ & $1.49 \times 10^{10}$ & $8.79 \times 10^{-3}$ & $8.31 \times 10^{33}$ & $6.34 \times 10^{43}$ \\
10 & $10^{-4}$ & $4.71 \times 10^{10}$ & $2.78 \times 10^{-3}$ & $8.31 \times 10^{33}$ & $2.00 \times 10^{44}$ \\
50 & $10^{-2}$ & $2.36 \times 10^{10}$ & $1.39 \times 10^{-1}$ & $2.06 \times 10^{35}$ & $2.43 \times 10^{45}$ \\
50 & $10^{-3}$ & $7.45 \times 10^{10}$ & $4.40 \times 10^{-2}$ & $2.06 \times 10^{35}$ & $7.70 \times 10^{45}$ \\
50 & $10^{-4}$ & $2.36 \times 10^{11}$ & $1.39 \times 10^{-2}$ & $2.06 \times 10^{35}$ & $2.43 \times 10^{46}$ \\
\hline
\end{tabular}

\subsection{Ray-tracing}

In our simulations, a scalar variable $\varphi$, as introduced in Saxton et al. (2001), is passively advected with the gas. Cells occupied purely by jet material are initially assigned the value $\varphi=1$; and the ambient medium initially has $\varphi=0$. Thus $\varphi$ traces the local fraction of matter originating in the jet.

We adopt the approximation of using the pressure field to trace the emissivity of the relativistic particles within jet plasma (e.g. Saxton et al. 2001). This approximation is based on the fact that the synchrotron emissivity, $j_{v}$ is proportional to $p B^{1+\alpha}$ where $p$ is the pressure, $B$ is the magnetic field and $\alpha$ is the spectral index. If the magnetic pressure, $B^{2} / 8 \pi$ tracks the particle pressure, then $j_{v} \propto p^{(3+\alpha) / 2}$, and $\alpha \approx 0.6$ typically. Even if the magnetic pressure does not track the pressure faithfully, it is likely that regions of high pressure still correspond to regions of high emissivity. 
We revolve the hydrodynamic data frames of $\varphi$ and $p$ to form 3D cylindrical structures. Using $\varphi$ to distinguish synchrotron-emitting jet material from gas originating in the background medium, the weighted emissivity is then $j_{v} \propto$ $\varphi p^{1.8}$. By integrating $j_{v}$ along rays through the $3 \mathrm{D}$ volume we generate surface brightness images of the jet and cocoon at a selected orientation. The resulting images are compared with observations in Sect. 5.

\section{Simulation results}

\subsection{General features}

The density distribution and velocity fields of representative frames from six of the simulations are shown in Fig. 3. At the beginning of each simulation, a bow shock is quickly established around the jet. The regions just downstream and inside the bow shock are dominated by the shocked dense interstellar medium (ISM). The innermost regions immediately surrounding the jet are occupied by a cocoon of low-density gas moving in a turbulent backflow opposite the jet direction. Flow velocities in some regions are comparable to the initial jet velocity $v_{\mathrm{j}}$, and are much larger than the bow shock velocity (see Fig. 4). Turbulent velocities are typically lower in the outer parts of the cocoon, further from the jet. The scalar tracer $\varphi$ (see Fig. 5) reveals that jet-derived matter dominates the inner regions of the backflow, whereas the outer regions just downstream of the bow shock are entirely composed of background gas.

The evolution of the backflow depends upon the left boundary condition. When the left boundary of the grid is open to outflow, the backflow occupies a cylindrical "sheath" surrounding and flowing opposite the jet, ultimately flowing off the grid. This "sheath" has a radius a few times that of the jet. It increases gradually as the jet progresses across the grid, but is insensitive to $\eta$. Very little of the surrounding gas mixes with backflowing jet plasma: most of the fast-moving backflow still exhibits high concentrations of jet plasma $(\varphi \gtrsim 0.5)$ when it exits the left boundary (see right column of Fig. 5).

In simulations with a closed, reflecting boundary, cocoon eddies accumulate inside a roughly conical volume, which is widest near the left boundary, and tapers towards the head of the jet. This "wake" cocoon expands laterally as the jet progresses forwards, and jet material accumulates and mixes with the shocked ISM. The eddies are typically smaller and have lesser concentrations of jet matter (lower $\varphi$ ) with increasing distance from the head of the jet (see upper panels of Fig. 5).

The density contrast of the jet, $\eta$, affects the gross structure of the surrounding radio emitting regions. If the left boundary is closed, jets with lower $\eta$ produce wider bow shocks and wider "wake" cocoons (see left panels of Fig. 3). However in cases with an open boundary the high- $\varphi$, fast backflowing "sheath" cocoon has approximately the same width, $\sim 4 r_{\mathrm{j}}-5 r_{\mathrm{j}}$, for all the values of $\eta$ we have studied: $\eta=10^{-2}, 10^{-3}$ and $10^{-4}$ (see right panels of Fig. 5). In such systems the bow shock widens marginally with decreasing $\eta$.

The amount of mixing between jet and ISM also shows a dependence upon $\eta$. For the open boundary mixing per unit length is more pronounced in the backflow for the larger values of $\eta$ (see the right panels of Fig. 5).

There is a similar dependence for the case of the closed boundary with some additional features. For $\eta=10^{-2}$, the cocoon widens almost linearly from the head of the jet (radius $\$ 3 r_{\mathrm{j}}$ ) and the degree of mixing in the backflow increases more or less gradually with distance from this point. However in cases with $\eta=10^{-4}$, the head of the jet is persistently surrounded by a broad (radius $\sim 6 r_{\mathrm{j}}$ ), low-density, high- $\varphi$ ellipsoidal region that is essentially the first turbulent cell in the backflow (see upper-left panels of Figs. 3, 5). There is a lot of mixing at the trailing edge of this cavity and average $\varphi$ values decline rapidly to the left of that region. Cases with $\eta=10^{-3}$ show an intermediate behaviour: the cavity is more extended in the $z$ direction and includes more fingers of entrained dense gas.

In the ultra-light $\left(\eta=10^{-4}\right)$ jets with an enduring cavity, the head of the jet surges back and forth along the axis between the front and back ends of the cavity. Similar surging behaviour occurs in most of the simulations, to different extents and at different frequencies. This cyclic process begins when turbulence in the backflow briefly compresses and constricts the jet at one point (see Fig. 7, panels 2 and 3). A portion of the front of jet is cut off, and its remnant mixes into the front of the cocoon. Jet plasma accumulates upstream of the obstruction (Fig. 7, panel 4), until it is able to surge through to a point much closer to the bow shock (Fig. 7, panels 5 and 6). Thus the terminal shock of the jet is not a constant feature: it may occur close to the bow shock (when the jet has just undergone a surge), or it may occur many jet diameters further upstream (when the jet is being disturbed by turbulence). The extent and frequency of surging depends on the jet parameters and the condition of the left boundary. In the closed-boundary, $\eta=10^{-4}$ cases, the strong, persistent, frontal eddy frequently pinches off the jet at the back end of the cavity, where the backflow converges back towards the surface of the jet. Cases with $(\eta, M)=\left(10^{-4}, 50\right)$ exhibit unusually extreme surging behaviour, because the relatively violent turbulence throughout the entire cocoon can pinch this ultra-light jet at almost any point along its length.

\subsection{Shocks and emission regions}

As discussed above (Sect. 3.2), the regions of strongest emission are high pressure shock features with a significant concentration of jet plasma (i.e. large $\varphi$ ). In agreement with past studies (Norman et al. 1982), we find several types of shocks characterising particular regions of the jet and its surroundings. The plots of $|\nabla p|$ in Fig. 16 show the shocks appearing as intense lines, in several regions of the flow.

We classify the different shocked regions as follows:

1. Firstly there is the bow shock driven by and encompassing the jet and its cocoon. The bow shock is persistent but is clearly not involved in the radio emission.

2. The strongest but most ephemeral shocks occur near the head of the jet where the jet deposits a large part of its energy and momentum. This feature pulsates back and forth 

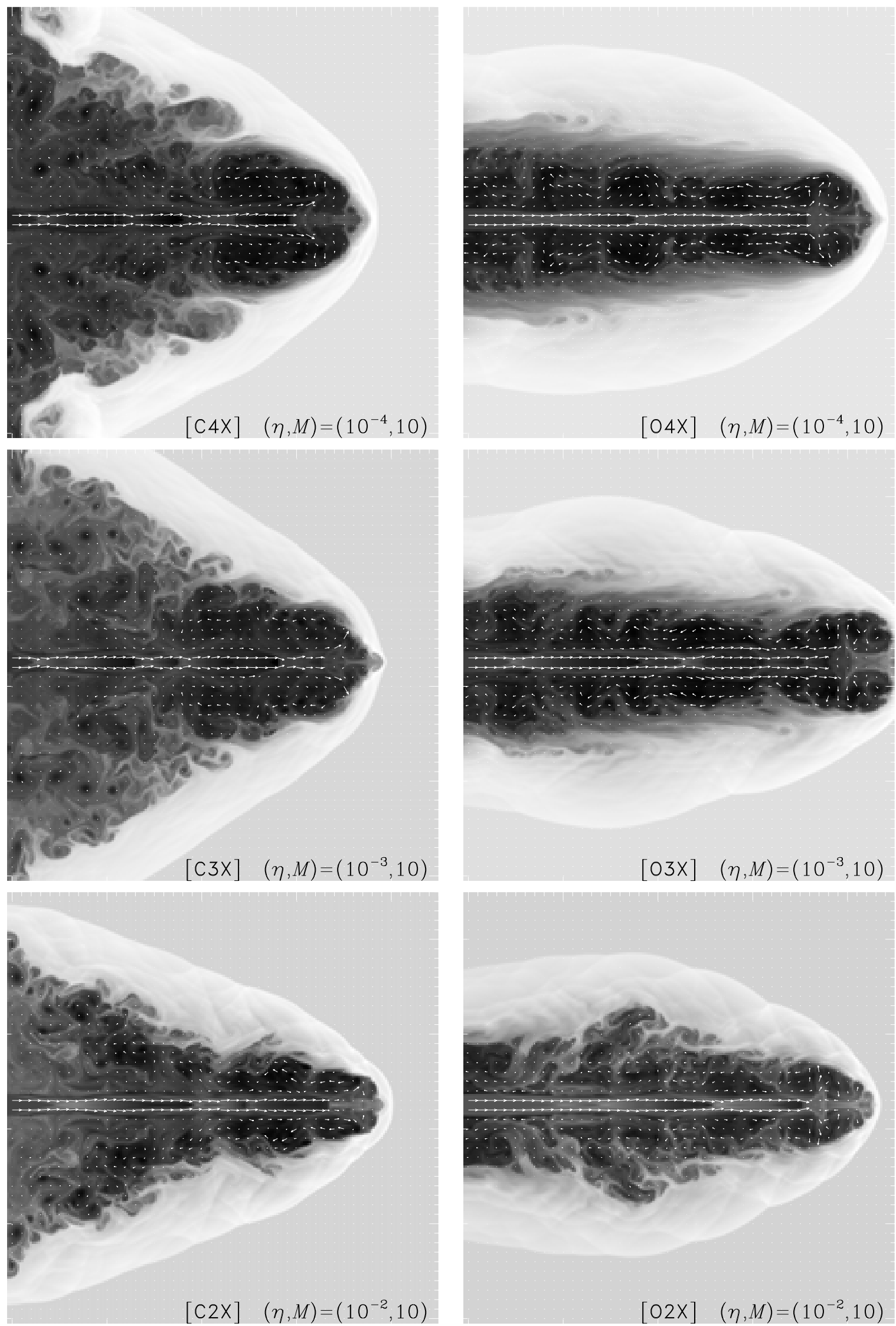

Fig. 3. Snapshots of flow velocity vectors superimposed on a $\log \rho$ image, in a $450 \times 300$ pixel zone $\left(0<z<7.5 x_{0}, r<2.5 x_{0}\right)$, at comparable stages of the jet's advance across the grid. Each row presents a different choice of $\eta$; in all cases $M=10$. The left and right columns show cases in which the left boundary is closed and open, respectively. 

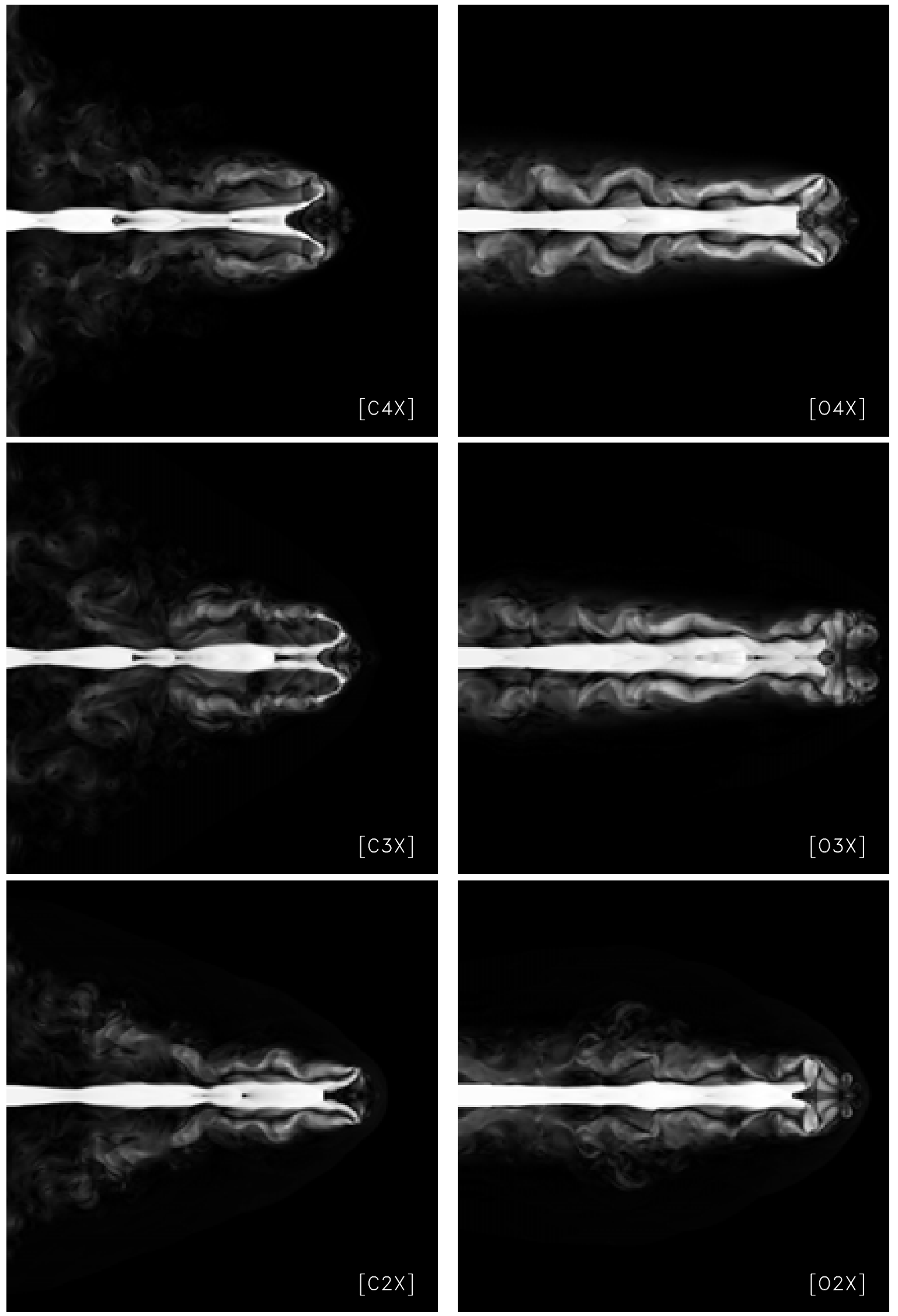

Fig. 4. Linearly scaled snapshots of the velocity magnitude, with the initial jet velocity $v_{\mathrm{j}}$ assigned to maximum brightness, for the same region, time and choice of parameters as in Fig. 3. 

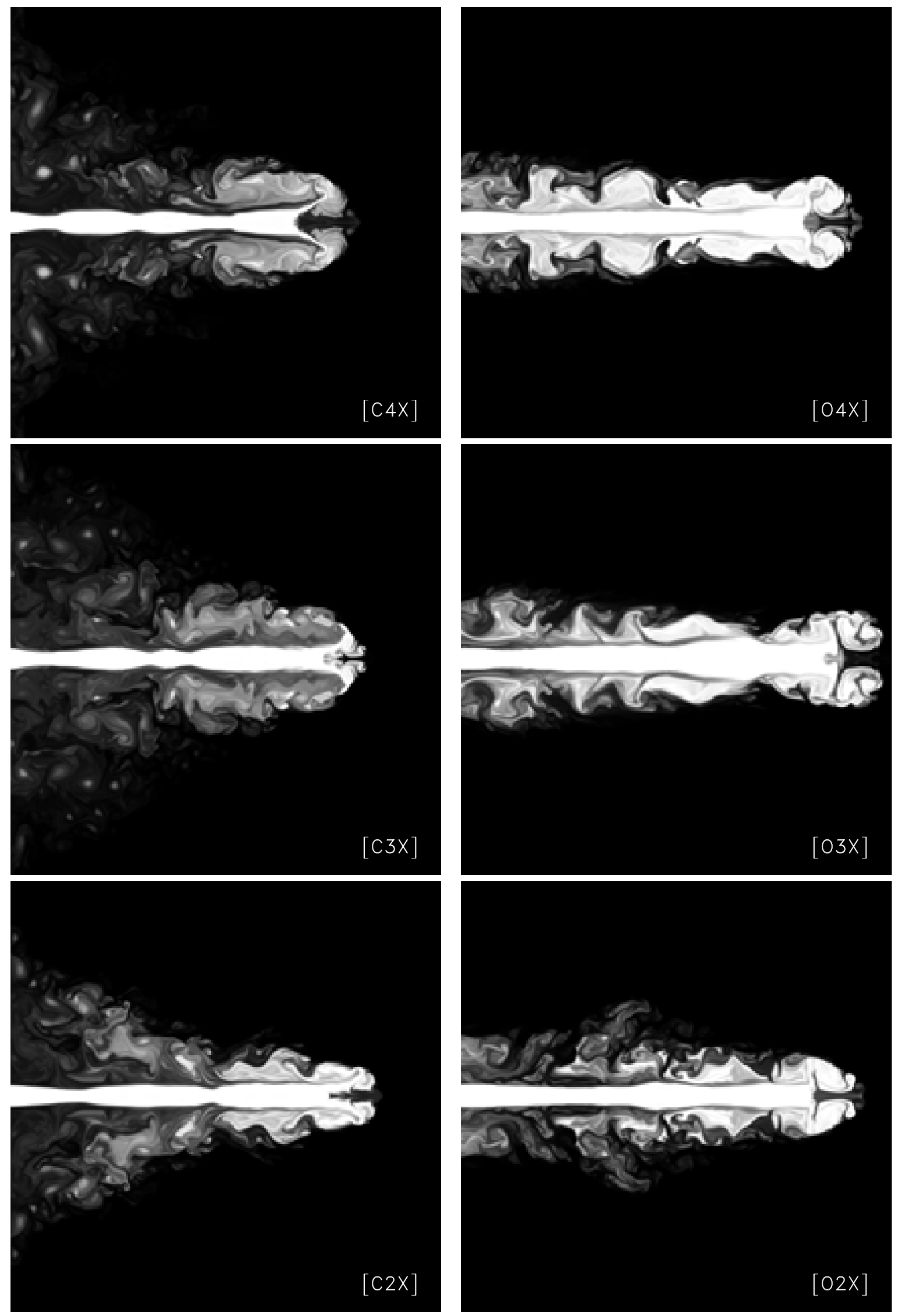

Fig. 5. Scalar tracer $\varphi$ showing the distribution of jet material for the same region, time and choice of parameters as in Fig. 3. White $(\varphi=1)$ represents pure jet plasma; black $(\varphi=0)$ represents gas originating in the ambient medium. 

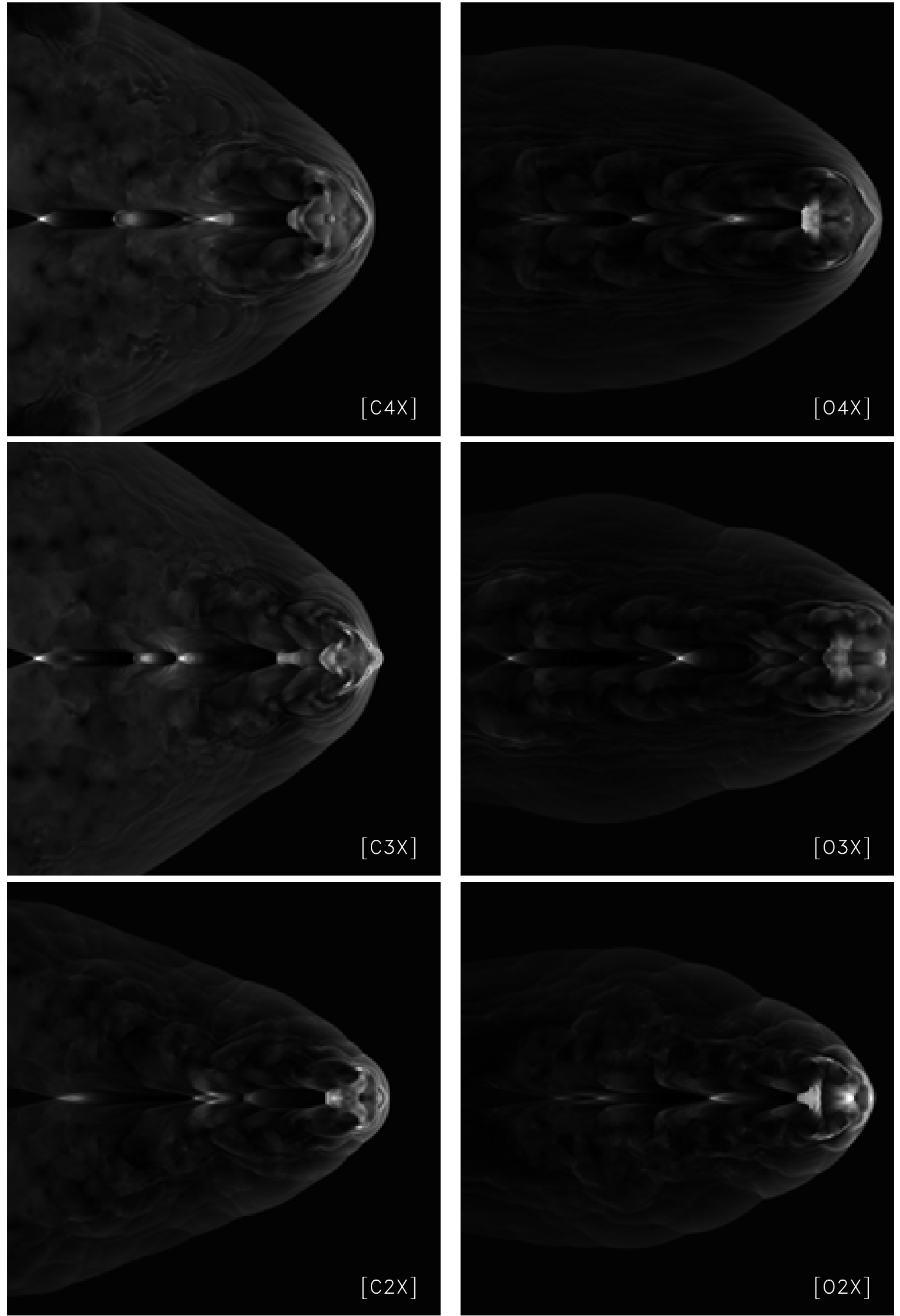

Fig. 6. Pressure maps corresponding to the frames in Fig. 3. 

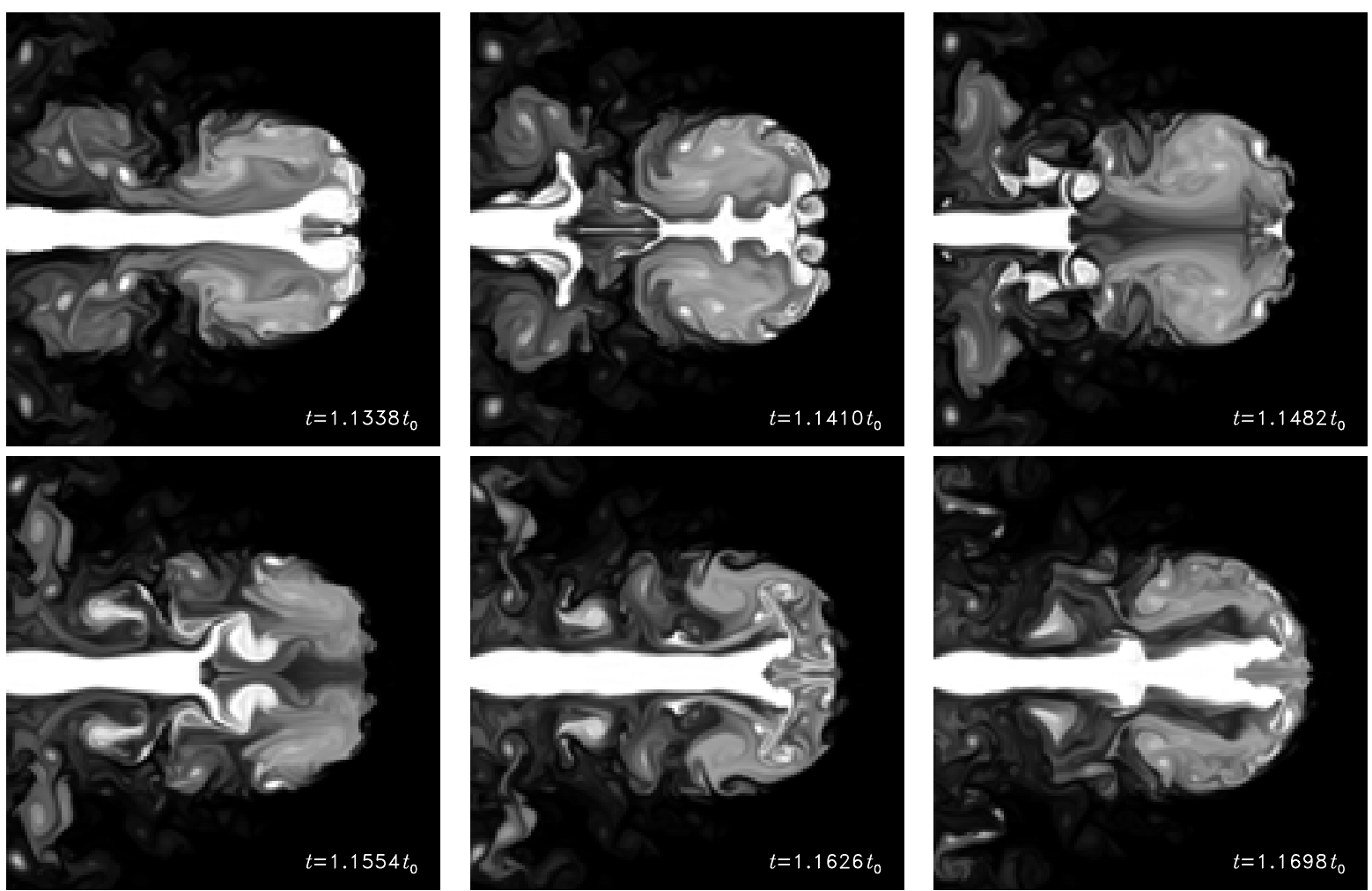

Fig. 7. Snapshots of the scalar tracer, $\varphi$, showing one episode of surging behaviour in simulation c4V, in which $(\eta, M)=\left(10^{-4}, 5\right)$. The spatial subregion is $\frac{5}{6} x_{0}<z<\frac{35}{6} x_{0}$ and $r<2.5 x_{0}$. White indicates pure jet plasma; black indicates a complete absence of jet plasma.

along the axis over distances several times the jet diameter, sometimes disappearing and reforming.

3. Shocks also propagate in the cocoon and either originate from near the head of the jet or are the result of local interactions between the jet, regions of the turbulent backflow and the surrounding layer of dense gas. Annular shocks are seen propagating both forwards and backwards through the backflow.

4. The jet itself is criss-crossed by diamond shocks caused by momentary and local compression by the turbulent backflow. As implied by the classical perturbation analysis of Birkhoff \& Zarantonello (1957), the diamond shocks are more widely separated in jets with greater $M$.

The distribution of $\varphi$ informs us which of the shocks produce significant synchrotron emission. Diamond shocks are always bright because they occur within pure jet plasma. For the same reason, the jet's terminal shock yields a prominent hot-spot in most frames of the simulations. However wider luminous structures are restricted to the extent of the high- $\varphi$ "sheath" cocoon in the systems with an open boundary, or the relatively unmixed frontal regions of the "wake" cocoons arising in the systems with a closed boundary. Thus, depending on the jet parameters and the boundary condition, there are no bright features exceeding $\sim 2 r_{\mathrm{j}}-7 r_{\mathrm{j}}$ in radius (see Fig. 5).

In summary, allowing for the axial symmetry of our simulations, the kinds of bright three-dimensional structures that are possible include: bright point-like, disk-like and cone-like shocks within the jet; in the backflow there may occur a diversity of rings and circular ribbons, with potentially flat, curved or solid cross-sections. In the following section, we consider how these structures may appear in two-dimensional projections onto the sky, and how these images may compare with observations.

\section{Relationship to Pictor A}

In this section we compare rendered images with the morphology of bright structures evident in the observations of Pictor A. The optical observations (Fig. 2) are a guide to the current positions of the shocks. Because of the longer lifetime of radio emitting electrons the radio image (Fig. 1) is indicative of the overall distribution of jet plasma within the cocoon.

In the rendering of our simulations we seek physical configurations that show a bright hot-spot at the front of the jet and a transverse bar upstream of the hot-spot. As refinements, we prefer images in which the bar appears brightened at its edges. The optical image (Fig. 2) also suggests faint arcs connecting the ends of the bar to the hot-spot. We also have in mind (in the optical data) the bright knot of emission at the intersection of the jet axis and the bar, and the faint tail of emission leading from that knot to the east.

We generated ray-traced images of each frame of each simulation, with the jet oriented at a variety of inclinations, $\theta=45^{\circ}, 60^{\circ}, 70^{\circ}, 80^{\circ}, 90^{\circ}(\theta$ is the angle between the jet and the line of sight). We inspected and compared all the resulting images and sorted them somewhat subjectively according to their consistency with the topology, proportions and brightness ratios seen in the Pictor A observations. In the following we consider the effects of orientation upon the quality of the 
matching rendered images, and the effects of the jet parameters. We also inspect the underlying physical nature of the bright features and their temporal evolution.

\subsection{Orientation}

The orientation of the jet has a substantial effect on the projected appearance of the axially symmetric luminous features surrounding the jet.

The hot-spot and knot are not decisive characteristics, since diamond and terminal shocks appear at numerous instances and their appearance is virtually independent of orientation. However the transverse bar in the Pictor A observations provides a tight constraint on the selection of appropriate rendered images. This elongated feature must be the projection of at least one luminous structure that is three-dimensionally extended. Therefore it is necessary but not sufficient to select snapshots that contain extended high-pressure features. When projected onto the sky at some orientation, only a subset of these configurations produce images similar to the observations.

When the jet is at a large angle to the plane of the sky, e.g. $\theta=45^{\circ}$, very few frames show the desired topology and brightness: typically a few times $0.1 \%$ of frames have a subjective resemblance to the images. The best images of jets at $45^{\circ}$ inclinations are shown in Fig. 8. In these cases, the appearance of a straight "bar," if any, is a coincidence due to the visual superposition of the near and far edges of unconnected rings in the backflow; it is not a single physical structure. One of the positive features of the better renderings at $\theta=45^{\circ}$ is the abundance of snapshots in which the hot-spot is projected in front of an edge of a bright ring in the backflow, thereby resembling the faint arcs connecting the hot-spot to the bar in the optical observations. However the occurence of conspicuously elliptical features throughout the image tends to ruin the overall resemblence, and the accidental bars appearing by superposition are never much wider than the hot-spot. Indeed the least-bad matches have small features compared to the initial jet, $r \lessgtr 2 r_{\mathrm{j}}$, and we speculate that the resemblences might be even less convincing if these structures could have been resolved more finely by the simulations.

When the system is viewed at inclinations $70^{\circ} \lesssim \theta \leq$ $90^{\circ}$, the matches are more abundant and better in quality. Approximately $4 \%$ of all frames rendered at $\theta=90^{\circ}$ show features qualitatively similar to the Pictor A observations. Approximately $3 \%$ of the frames at $\theta=70^{\circ}$ show the same quality of resemblance. Inclinations of $\theta=90^{\circ}$ produce images with a straight, bright transverse bar behind the hot-spot. Lower inclinations, progressively down to $\theta \approx 70^{\circ}$, yield more images in which arcs appear to connect the ends of the bar with the hot-spot. However the trade-off is that the apparent bar may be less straight and distinct.

At inclinations as low as $\theta=60^{\circ}$, frames that discernibly resemble observation are as rare as $\approx 0.6 \%$. Some of the best examples are presented in Fig. 9, but they are not compelling matches. Like the best morphological matches at $\theta=45^{\circ}$, their bar radii are $\$ 2 r_{j}$, and they tend to involve coincidental superposition of the far side of an annulus behind the hot-spot.
There are instances showing a favourable "pedestal" or "arcs" near the hot-spot, but the best simulated "bar" features are plainly elliptical rather than straight.

At a given inclination, good morphologies appear with roughly equal abundance for all choices of the jet parameters, with the exception of the extreme case $(\eta, M)=\left(10^{-4}, 50\right)$ where the turbulent backflow and the jet behave abnormally (see Sect. 5.2). For example renderings, see Figs. 10-15, and a selection of corresponding plots of $|\nabla p|$ in Fig. 16, which indentify the principal intense linear features with shocks. We display more examples for cases where $\eta=10^{-4}$ but this is only because these simulations produced more frames of data, and the emission features evolve more rapidly compared to the jet's advance across the grid (see Sect. 5.3 below).

As implied by the distribution of $\varphi$ and the commonplace occurrence of annular shocks throughout the backflow, the rendered snapshots include bright bars of diverse widths, (up to approximately $6 r_{\mathrm{j}}$ in cases with $\eta=10^{-4}$ ). The observed filament (Figs. 1, and 2) has a radius four times that of the hotspot. If the observed hot-spot corresponds to the width of the jet, then all of our simulations are in principle able to produce bright shocks in the backflow that are consistent in size with the observed filament.

\subsection{Brightness distribution}

Let us now consider the overall brightness distribution of various features in the image. This includes the brightness distribution of the filament, its brightness relative to the hot-spot and the overall brightness of the hot-spot and bar relative to the lobe. Note that in the observations, the brightest part of the filament is approximately $7 \%$ of the peak brightness in the hotspot, in the radio and about $2 \%$ in the optical.

Some frames rendered from the simulations have shapes that are consistent with the observations of Pictor $\mathrm{A}$, in terms of qualitative topology and approximate proportions, but may differ from the observations in terms of brightness. For instance, in some imperfect examples, the bar is dim on the edges rather than being edge-bright, e.g. $(\eta, M, t, \theta)=\left(10^{-4}, 10,0.6216 t_{0}, 90^{\circ}\right)$ (in centre panel, upper row of Fig. 11, and right column, Fig. 23) and $(\eta, M, t, \theta)=$ $\left(10^{-4}, 5,0.6221 t_{0}, 90^{\circ}\right)$ (centre panel, lower row of Fig. 11). In these cases, the bar is the image of a disk-like (rather than annular) shock that cuts the jet and a high- $\varphi$ transverse stream diverging from the jet.

In some cases in which the morphology is approximately correct, the ratio of filament to hot-spot brightness is larger than observed: e.g. $(\eta, M, t, \theta)=\left(10^{-2}, 10,0.296 t_{0}, 90^{\circ}\right)$ shown in the central panel of Fig. 14 ; and $(\eta, M, t, \theta)=$ $\left(10^{-2}, 10,0.8212 t_{0}, 90^{\circ}\right)$ (right column, Fig. 22$)$, in which the wide bar is a shock cutting through both the jet and cocoon. However, we cannot rule out cases with overly bright bars since relativistic beaming may be important in the hot-spot. There are also examples in which the ratio of filament to hot-spot brightness is approximately correct, e.g. the three examples in the uper row of Fig. 15. Here the brightness ratios are approximately $12 \%, 8 \%$ and $12 \%$ (ordered from left to right). 

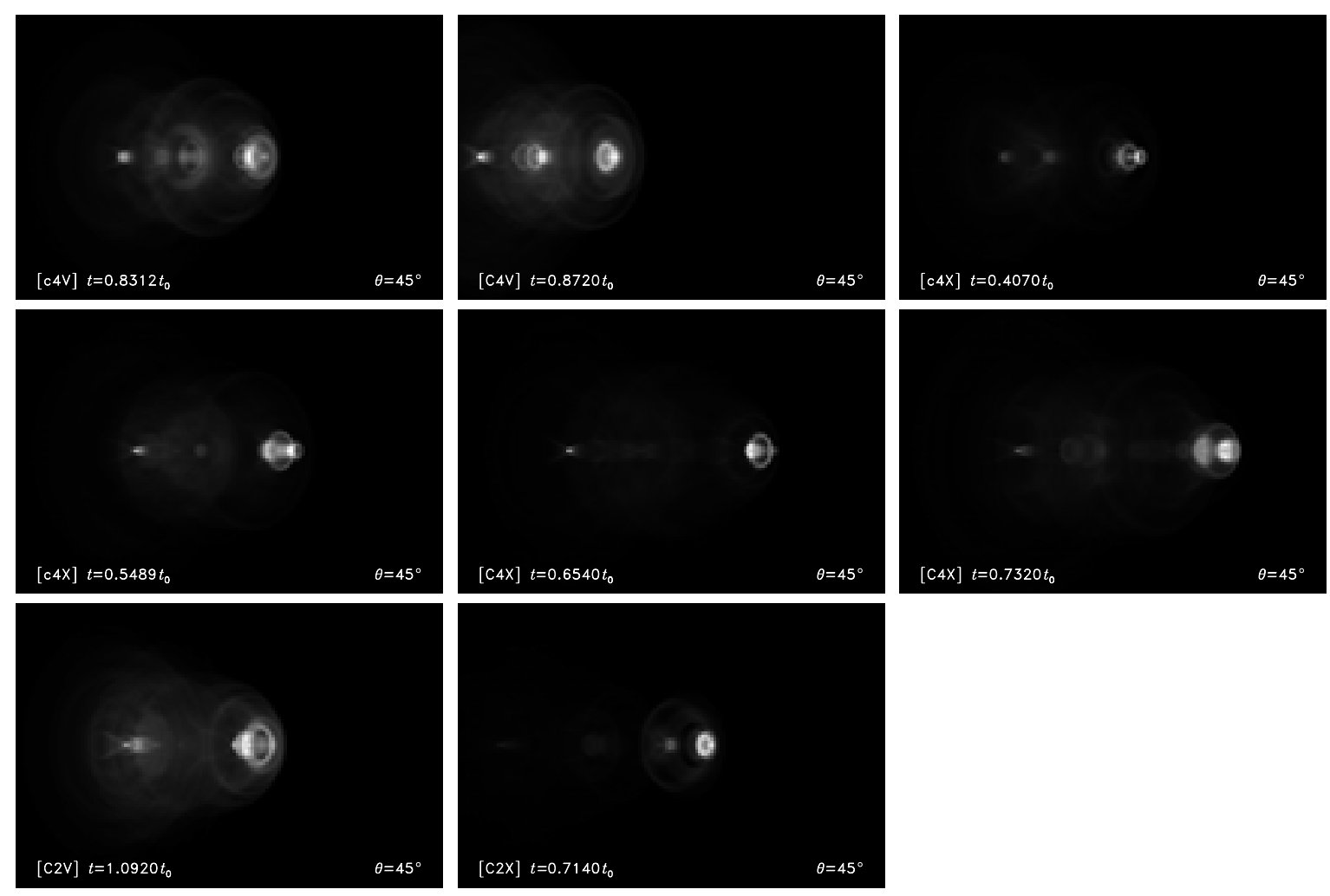

Fig. 8. $450 \times 300$ pixel raytraced images of a jet inclined at $\theta=45^{\circ}$ to the line of sight. None of them show an apparent transverse bar with a diameter much greater than that of the jet. The frontal "hot-spot" is a shock feature that is smaller than the initial diameter of the jet.

The appropriate ratios are preferentially found in the highest Mach number cases. A filament at the 7\% level is generally more difficult to find in a low Mach number lobe because of the lack of contrast with the background cocoon. The physics of the contrast between hot-spot and cocoon is discussed further below.

The observed filament is extended more to the south of the inferred jet than the north. Because of the axisymmetry of the simulations, this feature is not replicated. However, an axisymmetric approximation is justified in view of the very straight jet evident in the X-ray image (Wilson et al. 2001). Nevertheless, in most of the matches that we have presented in Figs. 10-15, the simulated filament is seen to be brighter towards the ends. This is the result of the annular shocks arising in the region of the hot-spot.

A feature of the observations that is replicated in approximately $40 \%$ of the matches in Figs. 10-15 is a small "knot" of emission at the centre of the bar. Such a feature is often the result of the superposition the bright annulus with a section of the jet that is also bright, such as a shock upstream of and related to the terminal shock of the hot-spot.

Yet another observational feature that appears in almost every panel in Figs. 10-15 is the radio knot south-east of the bar along the inferred direction of the jet. These knots correspond to diamond shocks in the jet; their exact location depends stochastically upon the details of the turbulence in the cocoon.

In the radio observations (Fig. 1), diffuse emission from the radio lobe in regions near the bar and hot-spot is $\lesssim 0.5 \%$ of the maximum surface brightness of the hot-spot. As Perley et al. noted, such a large contrast is highly unusual for a radio galaxy.
Ideally, our ray-traced images should produce a similar contrast between the brightness of bar and hot-spot and the wispy rings and dim emission pervading the rest of the cocoon.

Ideally, higher Mach number jets produce hot-spots with a higher contrast to the lobe. In simple terms, if the jet terminates in a single steady shock, the pressure contrast between the hot-spot and the cocoon is given by $p_{\text {hs }} / p_{\text {cocoon }} \sim M^{2}$. This aspect of jet physics lead Perley et al. to suggest that the Mach number may be as high as 40 . In terms of a realtivistic jet, this is equivalent to a Lorentz factor of 24 . However, the inferred parameters of the hot-spot (especially the pressure) could be significantly affected by beaming. The beaming pattern may be quite complex in view of the velocity field produced by the terminal oblique shock (see Sect. 5.4).

Ray-traced images from the simulations with parameters $(\eta, M)=\left(10^{-4}, 5\right)$ (see Fig. 10) give bar and hot-spot features that are typically only $\sim 10$ times brighter than the background cocoon. The brightness contrast is greater in simulations with $(\eta, M)=\left(10^{-4}, 10\right)$ (see Fig. 11). In this case the contrast varies bewteen $10^{1.3}$ and $10^{1.7}$. Simulations of very fast jets, (e.g. case C2L, with parameters $\left.(\eta, M)=\left(10^{-2}, 50\right)\right)$ can yield brightness contrasts in the range, i.e $\sim 10^{2-2.5}$ - consistent with the radio images of Perley et al. The tendency towards increasing contrast in the hot-spot and bar can be seen in the example contour maps of surface brightness for $\eta=10^{-2}$ and for the lower- $M$ cases of $\eta=10^{-4}$ in Fig. 17.

However this trend breaks in the case C4L where $(\eta, M)=$ $\left(10^{-4}, 50\right)$; the brightness contrast is exceptionally low - typically $\$ 30$ - compared to the more sharply defined features in the $(\eta, M)=\left(10^{-2}, 50\right)$ and $(\eta, M)=\left(10^{-3}, 50\right)$ simulations. 

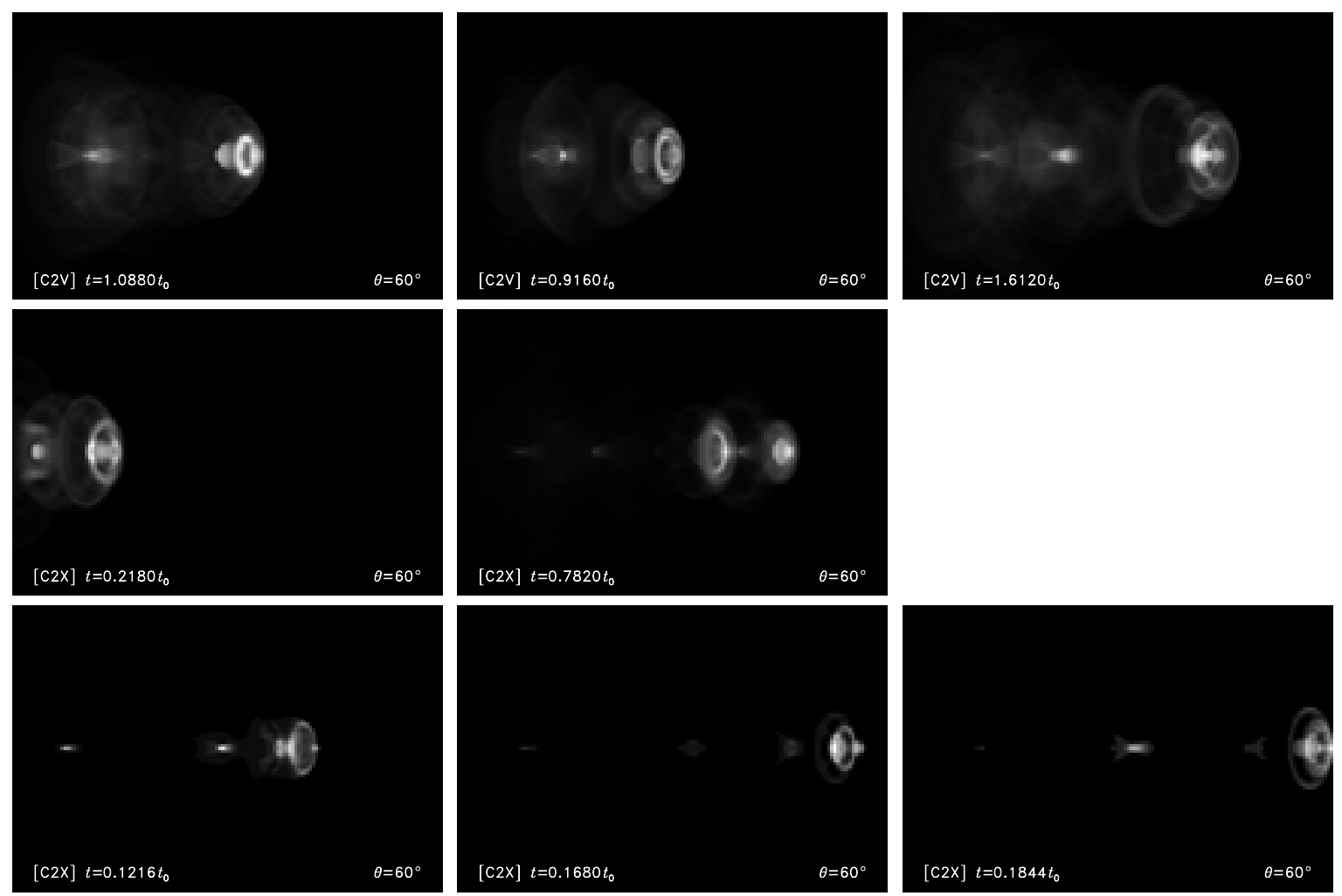

Fig. 9. Some of the best examples of raytraced images of a jet inclined at $\theta=60^{\circ}$ to the line of sight. The displayed subregions are the same size as those in Fig. 8.

This is an extreme manifestation of the surging behaviour that we have already noted. In this case the backflow turbulence is particularly vigorous and remains so at locations well behind the front of the bow shock. Strong eddies pinch off the extremely light jet at numerous locations along its length, and the jet's penetration into the cocoon varies dramatically within a dynamical timescale. Consequently, jet energy is deposited widely throughout the cocoon, rather than being concentrated at a quasi-stable head; moderately bright but transient rings and hot-spots occur throughout a large volume. This aspect may well be a feature of the restriction to axisymmetry causing the two-dimensional turbulence within the cocoon to be focussed on the jet.

\subsection{Temporal variation}

Physical configurations producing the desired morphology of surface brightness are ephemeral products of the jet's pulsating head and turbulent motions within the cocoon. It is therefore interesting to consider how these transient features vary in time. These considerations are also motivated by the potential of using hot-spot brightness ratios as a measure of relativistic beaming. Although these calculations are not relativistic we can examine the intrinsic distribution of hot-spot intensities. If it were to be the case, for example, that order of magnitude fluctuations in the ratio of hot-spot luminosities were to be likely, then the use of luminosity ratios as a measure of relativistic speeds within hot-spots would be limited. We address this point below following consideration of the structure function of individual hot-spots.

The dynamical timescale of structures in the jet material, such as the hot-spot and shocks in the jet and backflow, characteristically depends upon the jet's diameter and internal sound speed,

$t_{\mathrm{dyn}} \equiv \frac{2 r_{\mathrm{j}}}{c_{\mathrm{s}, \mathrm{j}}} \approx 0.39 \sqrt{\eta} t_{0}$

Thus $t_{\text {dyn }} \approx 0.04 t_{0}$ for jets with $\eta=10^{-2}$, and $t_{\text {dyn }} \approx 0.004 t_{0}$ for jets with $\eta=10^{-4}$. Since the brightest feature is usually a terminal hot-spot or some other similarly compact structure, we expect the instantaneous peak surface brightness to vary in time on a time scale on the order of magnitude of $t_{\mathrm{dyn}}$. Indeed this is the case, as shown by the structure functions (Simonetti et al. 1985) of the time-variation of the peak brightness of images rendered at $\theta=90^{\circ}$ (see Fig. 18). The structure functions were calculated from a time series based upon the peak intensity in each frame divided by the long term mean. (Frames containing initial transients were omitted.) Hence the amplitude of the structure function gives one an estimate of the square of the relative variabiliity on the relevant timescale.

On time scales longer than $\sim t_{\text {dyn }}$ the behaviour of most structure functions is effectively white noise (power index $\approx 0$ ); whereas the behaviour is like "red noise" on shorter timescales. The transition timescale between these behaviours is of order $0.1-0.5 t_{\text {dyn }}$ and depends little on $M$. However jets with greater $M$ generally show greater relative variability. 

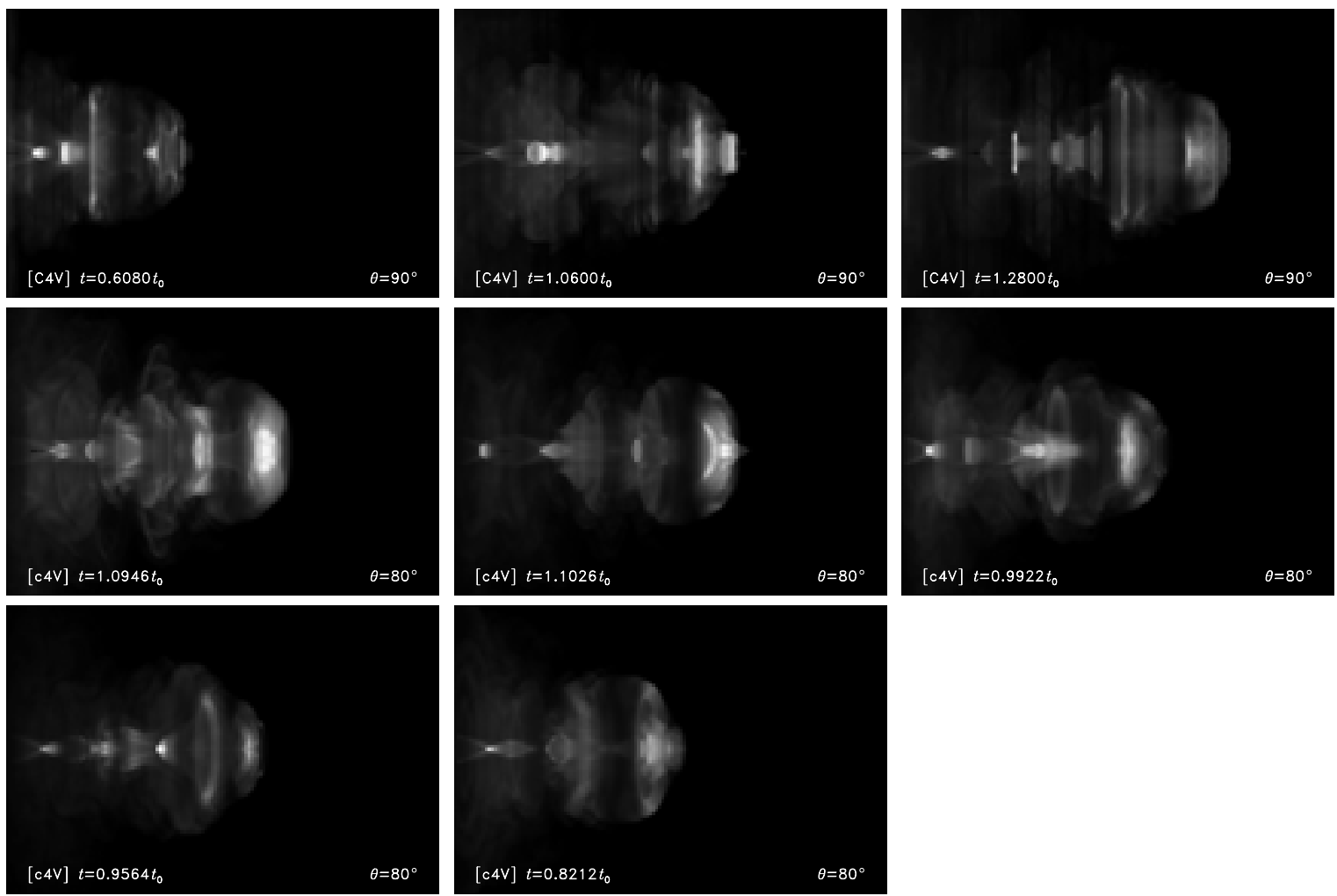

Fig. 10. A selection of morphological matches to Pictor A, from ray-traced renderings in a $450 \times 300$ pixel sub-region about a jet with parameters $(\eta, M)=\left(10^{-4}, 5\right)$.
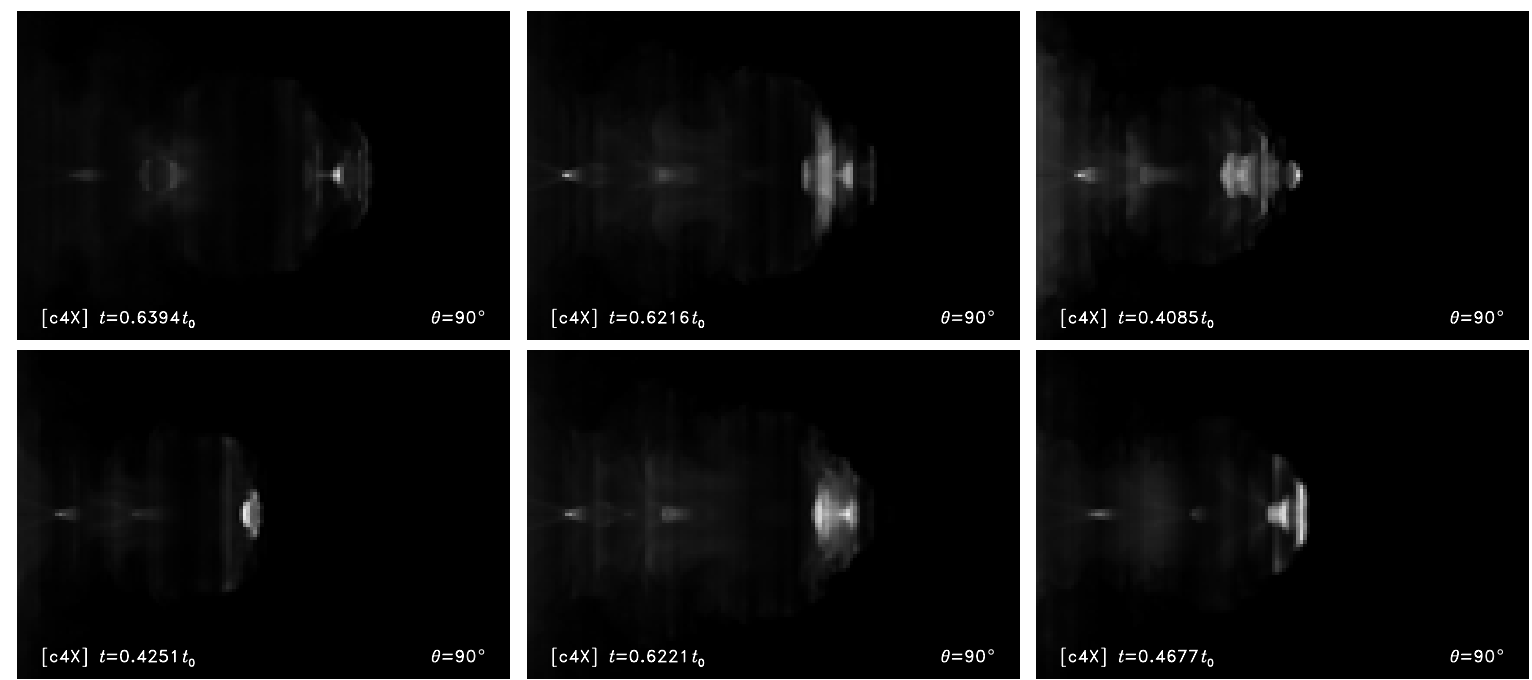

Fig. 11. Morphological matches as in Fig. 10 but with jet parameters $(\eta, M)=\left(10^{-4}, 10\right)$.
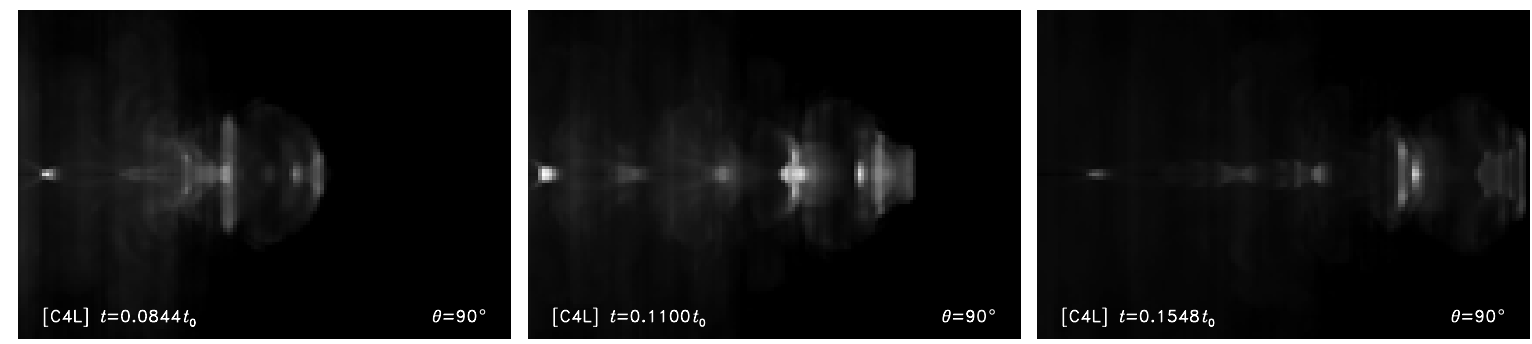

Fig. 12. Morphological matches as in Fig. 10 but with jet parameters $(\eta, M)=\left(10^{-4}, 50\right)$. 

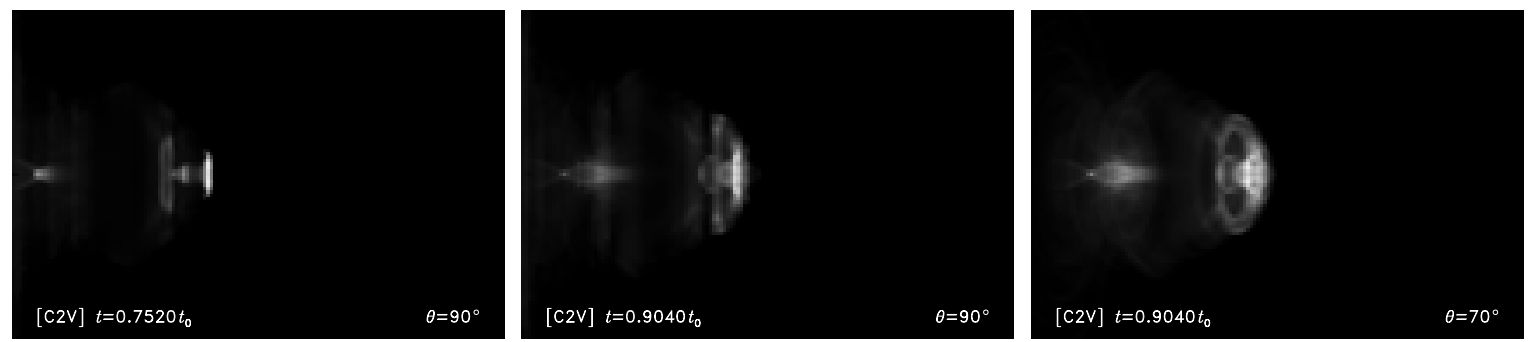

Fig. 13. Morphological matches as in Fig. 10 but with jet parameters $(\eta, M)=\left(10^{-2}, 5\right)$.
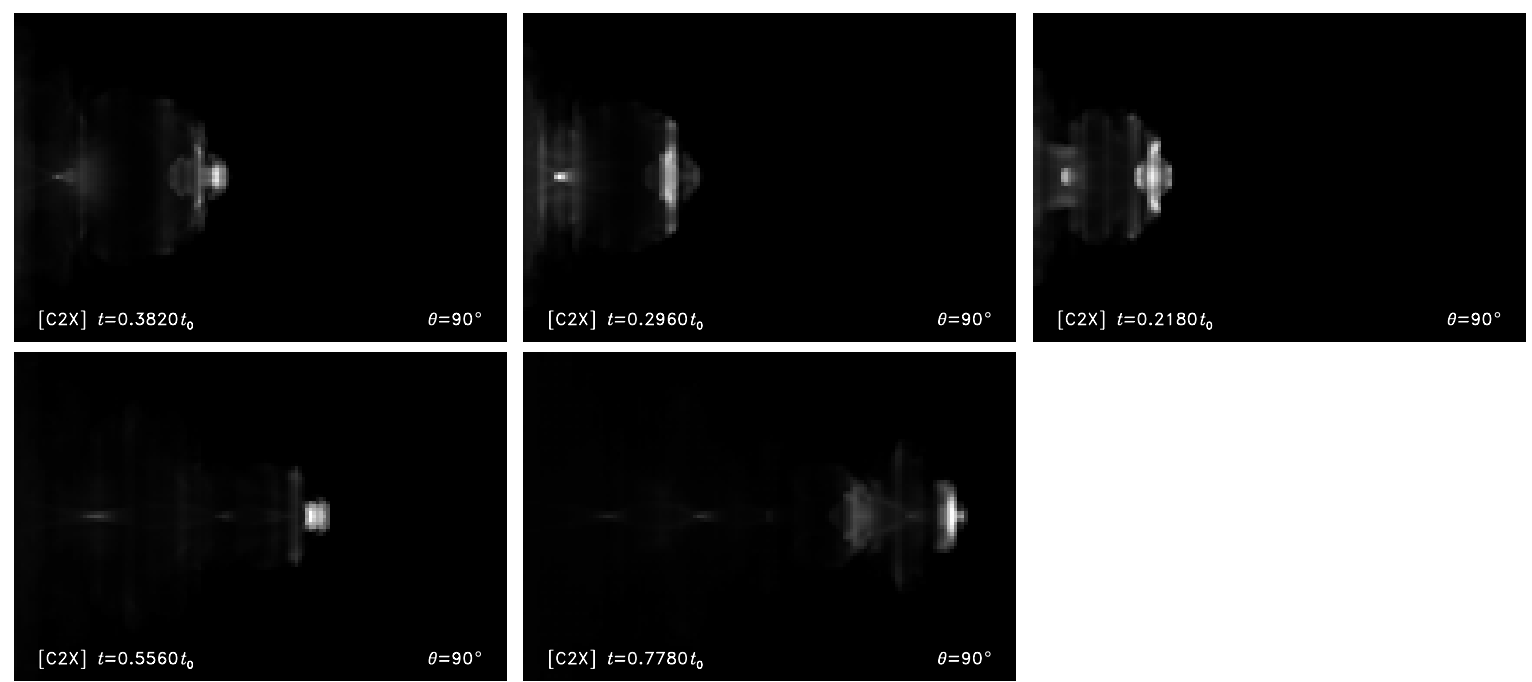

Fig. 14. Morphological matches as in Fig. 10 but with jet parameters $(\eta, M)=\left(10^{-2}, 10\right)$.
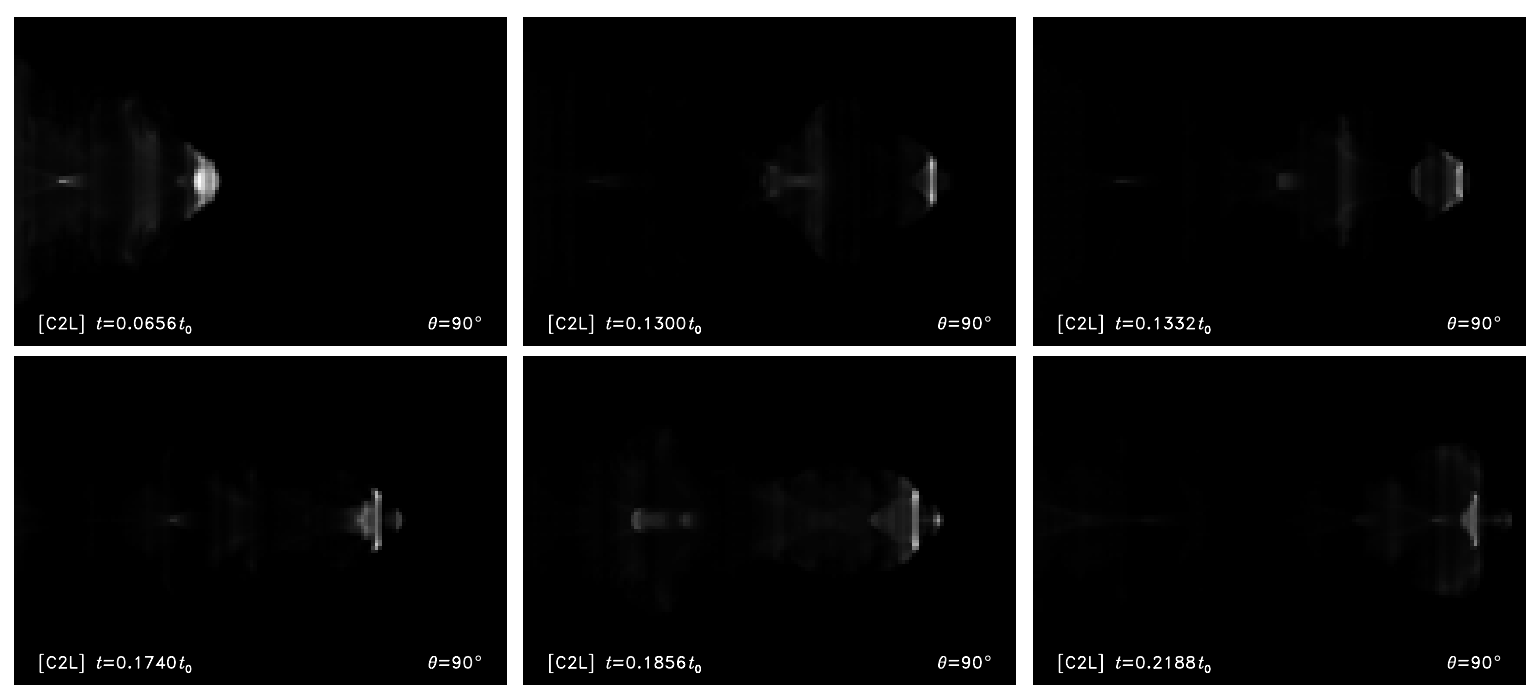

Fig. 15. Morphological matches as in Fig. 10 but with jet parameters $(\eta, M)=\left(10^{-2}, 50\right)$.

The one exceptional case is the $(\eta, M)=\left(10^{-4}, 50\right)$ simulation with the closed boundary. In this simulation there is long term evolution (evident in the upturned curve at long timescales in the upper left panel of Fig. 18) related to the highly turbulent cocoon upon which we have already commented in Sect. 5.2.

In all cases bar the exceptional case, the left boundary condition has negligible effect on the variation of peak surface brightness (compare left and right columns of Fig. 18).
This is the result of the proximity of the brightest structures to the terminal jet shock, rather than parts of the flow directly affected by the left boundary.

In physical units, with $t_{0} \sim 10^{7} T_{7}^{-1 / 2} \mathrm{yr}$, the dynamical timescale is $t_{\mathrm{dyn}} \sim 4 \times 10^{5(4)} \mathrm{yr}$ for a jet with $\eta=10^{-2(4)}$. These time-scales are much shorter than the time taken for light or jet plasma to traverse the $2.1 \times 10^{2} / h \mathrm{kpc}=6.4 \times 10^{5} / h$ ly projected distance between the nucleus and either hot-spot. Therefore the intrinsic flickering of two opposite hot-spots are causally 

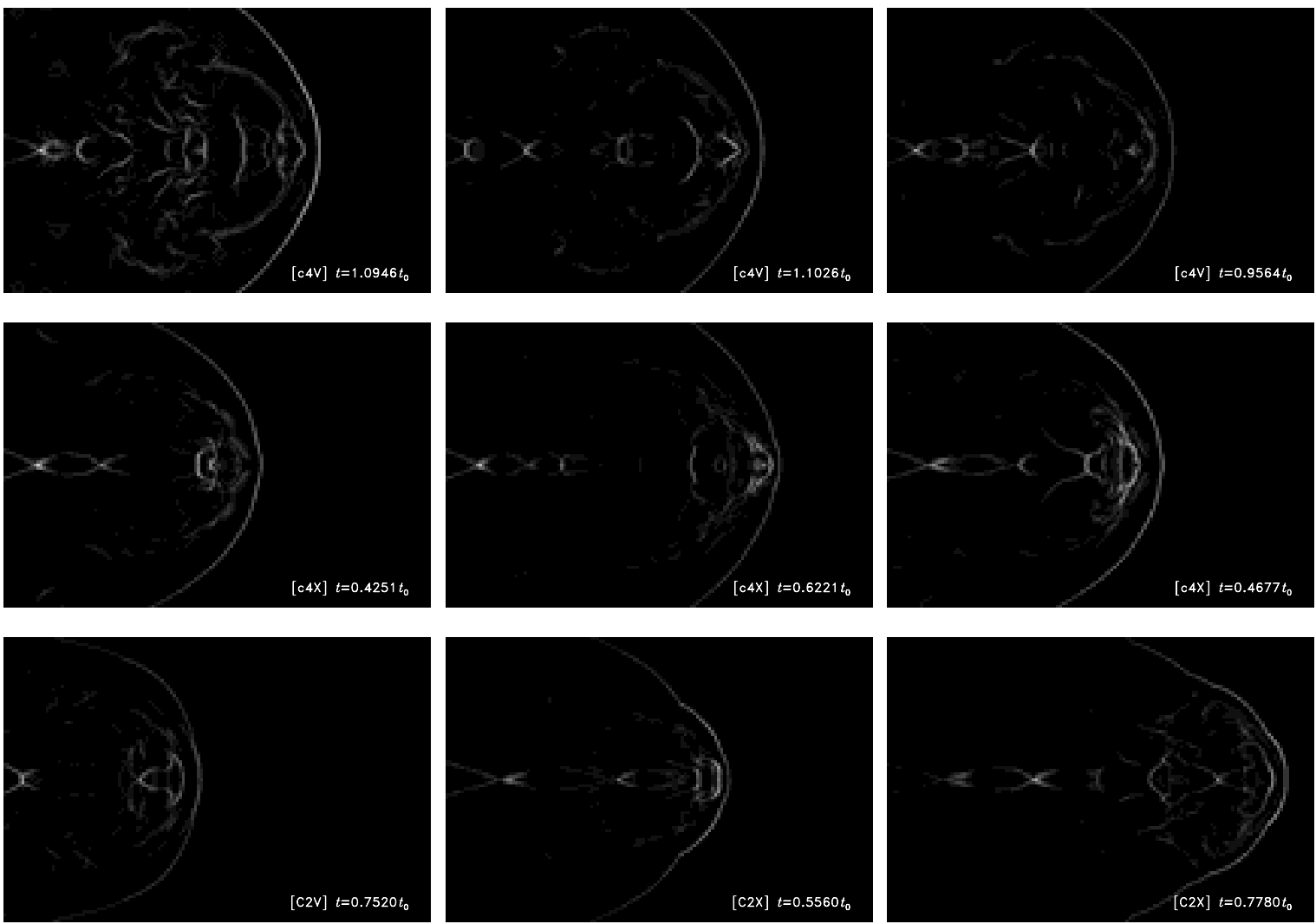

Fig. 16. Plots of $|\nabla p|$, delineating the presence of shocks. Panel dimensions are the same as in Figs. 10-15.

independent. Figure 19 shows the cumulative distribution function of the ratio of the peak brightness of one spot with respect to the other, $R=I_{1} / I_{2}$, calculated from simulation output according to the derivation in Appendix A. The ratio has a more extended distribution in cases with larger Mach number, consistent with the larger amplitude of the structure function. For a given choice of jet parameters $(\eta, M)$, an open boundary condition gives rise to substantially greater variability. For instance, the probability that $R>4$ or $R<\frac{1}{4}$ (see Table 3) is approximately $20 \%$ for cases with $M=50$ and an open boundary but only ranges from $\approx 4-13 \%$ in the cases we studied with a closed boundary. Likewise for small Mach number cases, for example with $(\eta, M)=\left(10^{-3}, 5\right)$ the probability that $R>4$ or $R<\frac{1}{4}$ ranges from $3-7 \%$ with an open boundary but only $0.4-1.5 \%$ with a closed boundary. Thus in cases where the jet has a low Mach number, the relative brightnesses of hot-spots on different sides of a source is a reasonable measure of nonintrinsic effects, including relativistic beaming, however intrinsic variability may be significant if the Mach number is large $(M \gtrsim 10)$. In general terms these distributions provide a means of quantifying the uncertainty in using hot-spot ratios in various applications.

\subsection{Underlying structure and activity}

The reproduction of images resembling observations of the western hot-spot of Pictor A requires a strong shock on the jet axis, and some other, wider shock structure in high- $\varphi$ regions
Table 3. Probability of a hot-spot ratio of at least $4: 1, \operatorname{Pr}(R \geq 4)+$ $\operatorname{Pr}\left(R \leq \frac{1}{4}\right)=2 F\left(\frac{1}{4}\right)$, occuring at any instant as a result of intrinsic variability, calculated from representative time sequences of simulations that are finely resolved in time (frame interval $\ll t_{\mathrm{dyn}}$ ).

\begin{tabular}{cccccccccc}
\hline \hline left B.C. & $\eta$ & $M$ & $2 F\left(\frac{1}{4}\right)$ & & & left B.C. & $\eta$ & $M$ & $2 F\left(\frac{1}{4}\right)$ \\
\cline { 1 - 4 } closed & $10^{-4}$ & 5 & 0.004 & & open & $10^{-4}$ & 5 & 0.065 \\
closed & $10^{-4}$ & 10 & 0.027 & & open & $10^{-4}$ & 10 & 0.183 \\
closed & $10^{-4}$ & 50 & 0.055 & & open & $10^{-4}$ & 50 & 0.206 \\
closed & $10^{-3}$ & 5 & 0.009 & & open & $10^{-3}$ & 5 & 0.026 \\
closed & $10^{-3}$ & 10 & 0.068 & & open & $10^{-3}$ & 10 & 0.144 \\
closed & $10^{-3}$ & 50 & 0.049 & & open & $10^{-3}$ & 50 & 0.197 \\
closed & $10^{-2}$ & 5 & 0.015 & & open & $10^{-2}$ & 5 & 0.034 \\
closed & $10^{-2}$ & 10 & 0.107 & & open & $10^{-2}$ & 10 & 0.076 \\
closed & $10^{-2}$ & 50 & 0.128 & & open & $10^{-2}$ & 50 & 0.173 \\
\hline
\end{tabular}

of the backflow. However more than one kind of physical situation can produce an appropriate image. Here we consider the particular instantaneous arrangements of shocks and jet plasma that yield some of the example images selected and displayed in Figs. 10-15. Examples of the related dynamics are given in Figs. 20-24, in which the panels show simulated intensity maps along with corresponding snapshots of pressure, $\varphi$ and density with overlaid velocity vectors. (In referring to these figures we use $\mathrm{L}$ for left and $\mathrm{R}$ for right panels respectively.) 

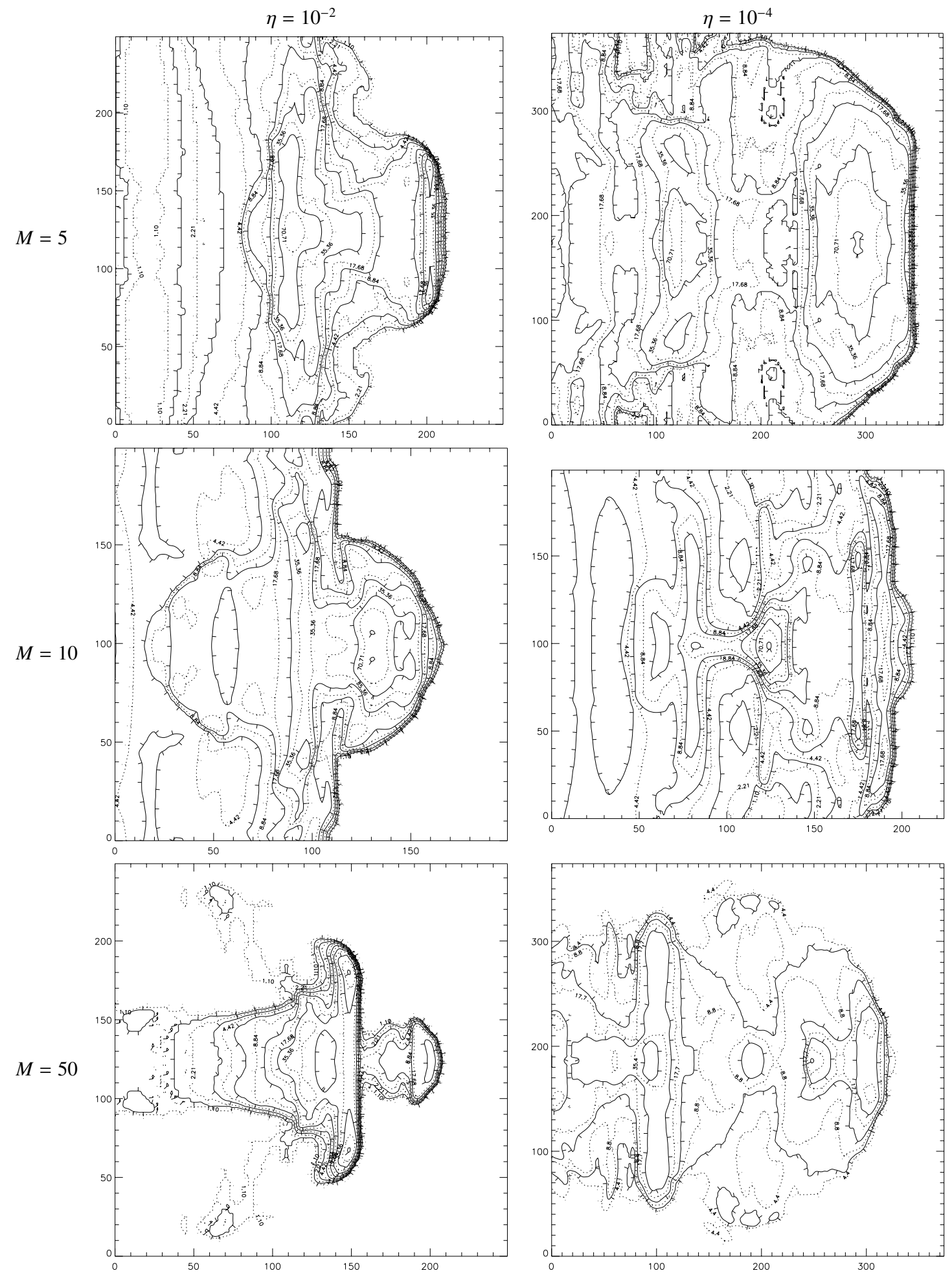

Fig. 17. Ray-traced surface brightness contour maps of the hot-spot vicinity, selected from the simulations with a closed left boundary. Contour levels are at consecutive multiples of $1 / \sqrt{2}$ times the peak value. Each column shows a different case of $\eta$; each row corresponds to a different $M$. For the sake of clarity, contour lines are alternately dotted and solid, and show normal marks directed along the "downhill" gradient.

In practice, a bar and hot-spot may arise from different underlying structures at any stage of the jet's surging behaviour. A hot-spot is commonly the termination shock at the head of the jet. However amongst our $\eta=10^{-4}$ selections, there are some cases such as Fig. 20L, 21L, 21R, and the detailed case
Figs. 25-27 where the head of the jet is at the back of the leading cavity, and not directly connected to the hot-spot. In such cases the forward hot-spot consists of a high pressure region of moderately mixed jet and thermal gas, or is an isolated blob of high- $\varphi$ matter previously separated from the jet. 

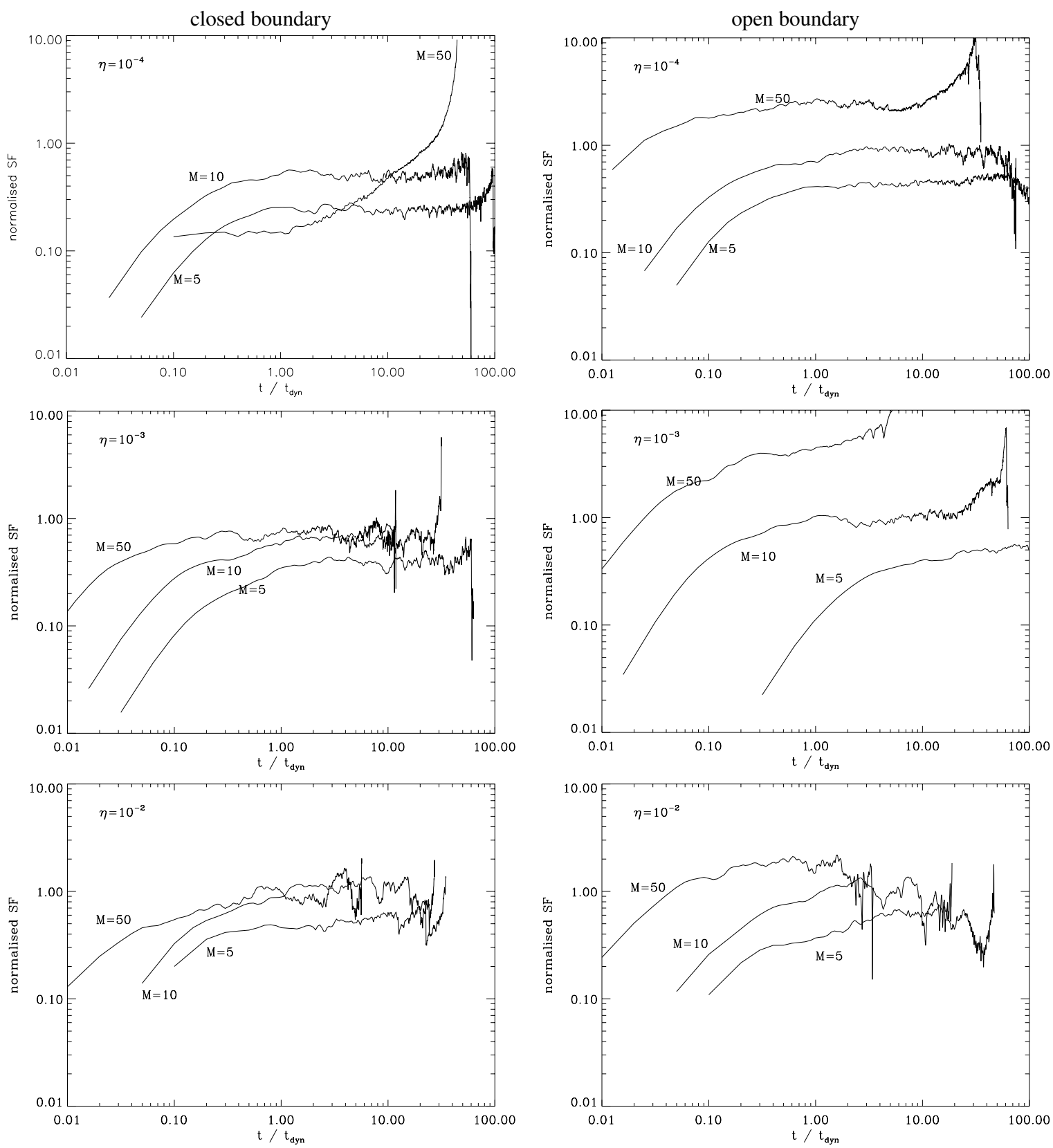

Fig. 18. Normalised structure functions evaluated for flickering of the peak intensity of the emission regions. The left and right columns show different cases of the left boundary condition. For comparison of relative variability, the raw time series were normalised to the respective mean intensities. We excluded data points from during the early establishment of the cocoon and the eventual exit of the cocoon and jet through the right boundary.

A bar may result from a variety of different kinds of annular or disk-like structures. In some cases (Figs. 20L, 20R, 24L) the bar is a broad shock at the head of the jet, or a body of jet plasma near the head. In some cases, it is the shocked rim of a funnel-shaped or disk-shaped volume of plasma that has split off the head of the jet but yet remains largely unmixed with the thermal gas in the cocoon (see Figs. 21R, 22R, 23R, 25-27). In other instances, the bar is a wide annular region in a part of the backflow well separated from the head of the jet, when such a region either preserves a moderate or high concentration of jet plasma, or experiences a strong shock locally (see Figs. 22R, 21L).

There are cases in which an ideal bar and hot-spot are formed, but additional features spoil the resemblance to Pictor A. For example, in one selection at $t=0.4085 t_{0}$ (Fig. 23L; or upper right panel of Fig. 11) the bar and hot-spot are high- $\varphi$ shocks in the vicinity of the jet's head, but an additional strong reverse shock in the jet appears as another bright feature to the left of the bar. Such reverse shocks are an occasional by-product of the activity that produces a hot-spot as a 

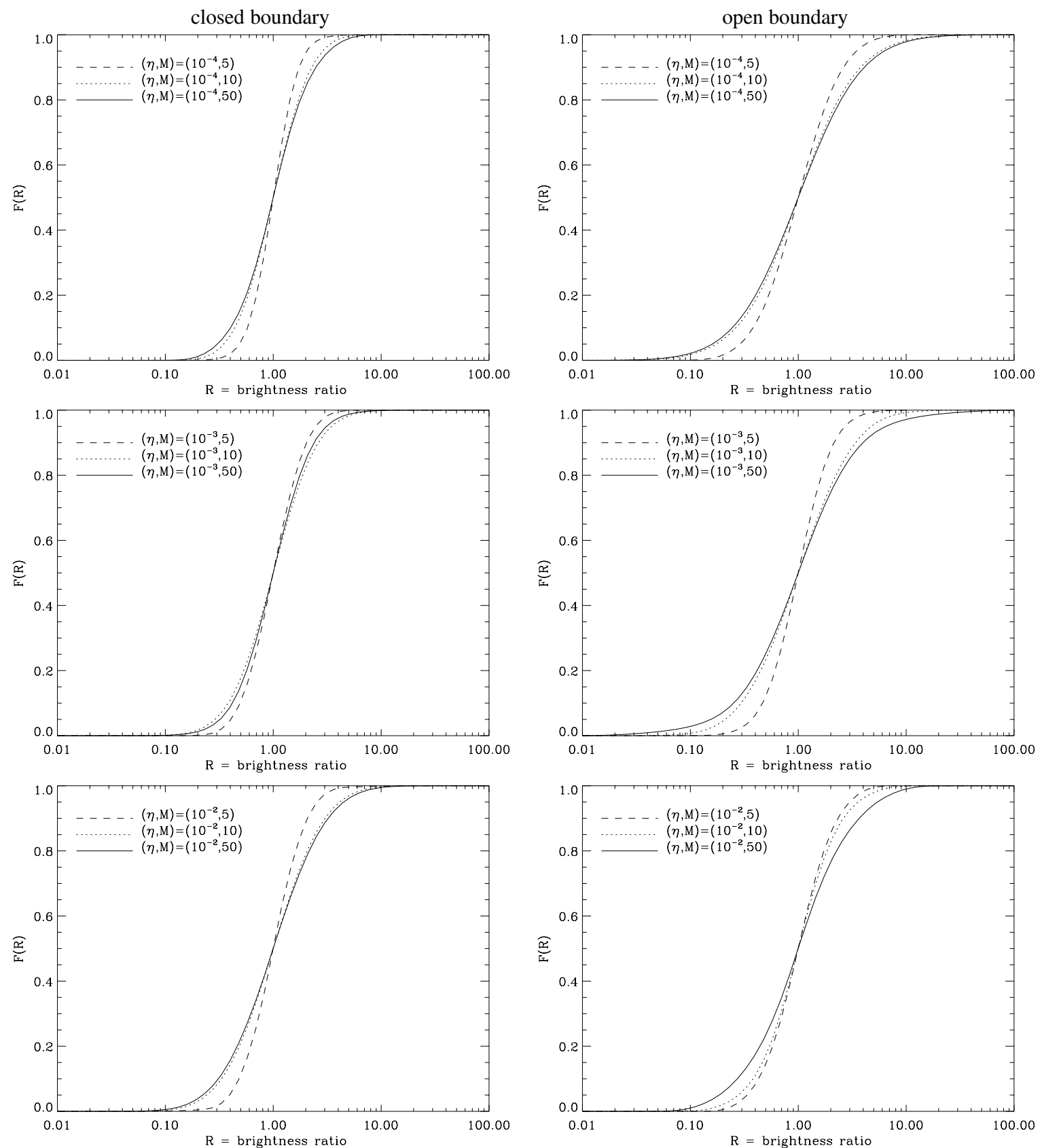

Fig. 19. Cumulative probability distribution functions, $F(R)=\operatorname{Pr}\left(R^{\prime} \leq R\right)$, for the ratio of peak surface brightnesses of two hot-spots which are flickering independently. Left and right columns respectively show cases with closed and open left boundaries. Within each panel, the three curves correspond to cases of $M=5,10,50$ for dashed, dotted and solid lines respectively.

forward shock and annular shocks propagating into the backflow. Milder versions of the reverse shock may give the appearance of the "knot" or "pedestal" observed on the jet axis.

It is not possible within the space of one paper to demonstrate the physical details underlying the evolution of all the physical configurations that give the appearance of a bar and hot-spot. In order to describe one particular process for the formation of a bright hot-spot and bar, we show in Fig. 25, a time sequence of linearly scaled pressure images from the closedboundary, $(\eta, M)=\left(10^{-4}, 5\right)$ simulation with fine time resolution (simulation $\mathrm{c} 4 \mathrm{~V}$ ). The first frame is at $t=0.9952 t_{0}$ and subsequent frames are at intervals of $0.0006 t_{0}$ numerical time units. Figure 26 shows corresponding frames of the distribution of $\varphi$. Figure 27 shows rendered images of the surface brightness at $\theta=90^{\circ}$. Figure 28 shows corresponding flow velocity fields superimposed upon $\log \rho$ greyscale images.

At the beginning of the sequence (frame 1), we see the usual haze of emission from weak shocks in the $\varphi \approx 0.5$ cavity at the head of the cocoon. A bright hot-spot appears at the front of the cavity and this is a high-pressure, shocked region with $\varphi \approx 1$, left behind by the head of the jet when it was previously at the foremost extremity of its surging motion. 

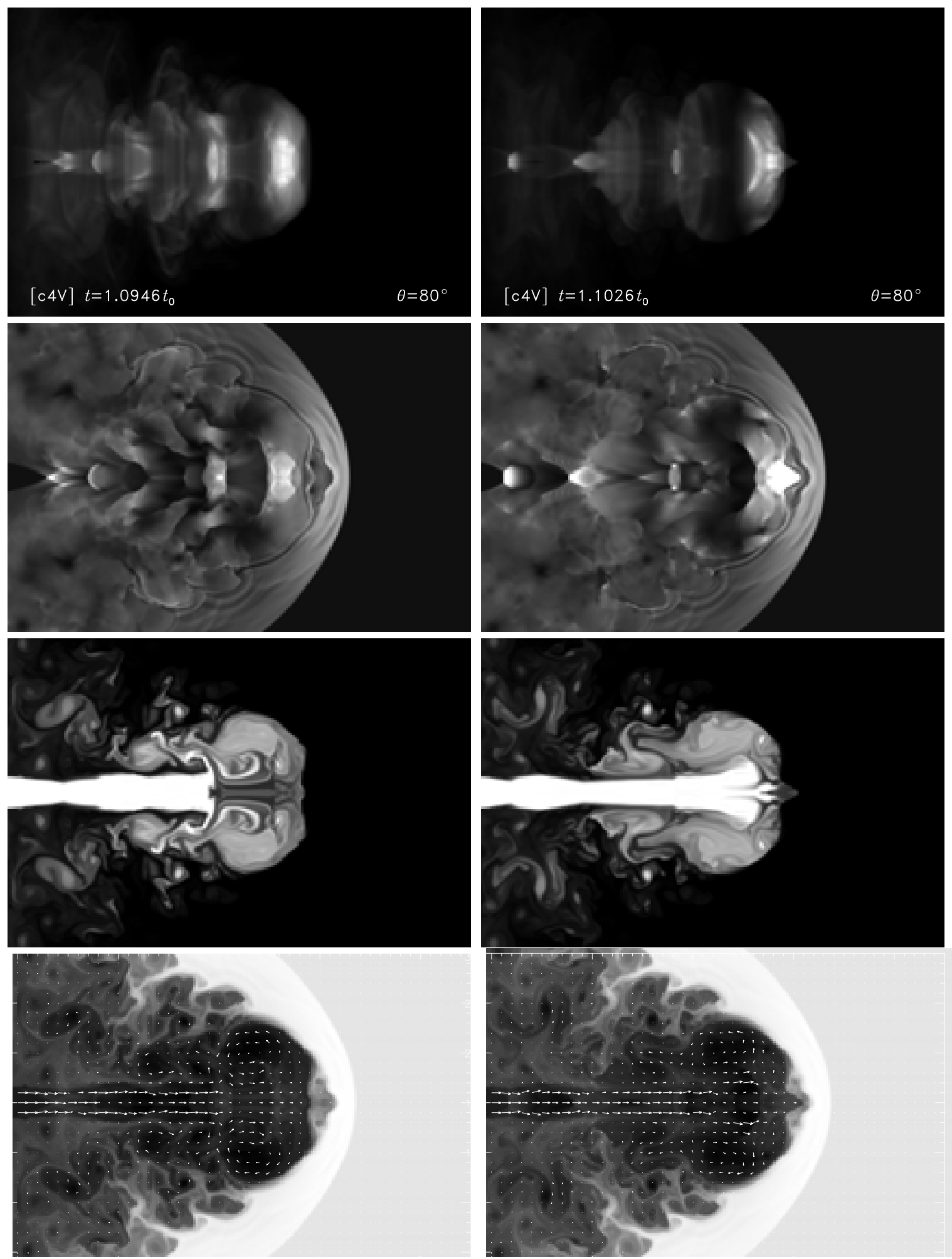

Fig. 20. $450 \times 300$ pixel sub-region around a jet with parameters $(\eta, M)=\left(10^{-4}, 5\right)$. Rows from top to bottom show: raytraced images; pressure; the tracer of jet material, $\varphi$; and logarithmic density plots overlain with flow velocity vectors.

In this frame, the head of the jet is towards the rear of the cavity. The jet splits at a terminal shock, dividing into a funnel-shaped structure consisting of unmixed jet plasma moving in a radially outwards, positive- $z$ direction, at speeds comparable to $v_{\mathrm{j}}$. The outermost edge of this structure is curled back upon itself, and trails off into the backflow closest to the surface of the jet. The region between this backflow and the inner surface of the funnel is underpressured compared to average conditions in the cocoon, however the outer lip of the funnel is a high pressure surface. 

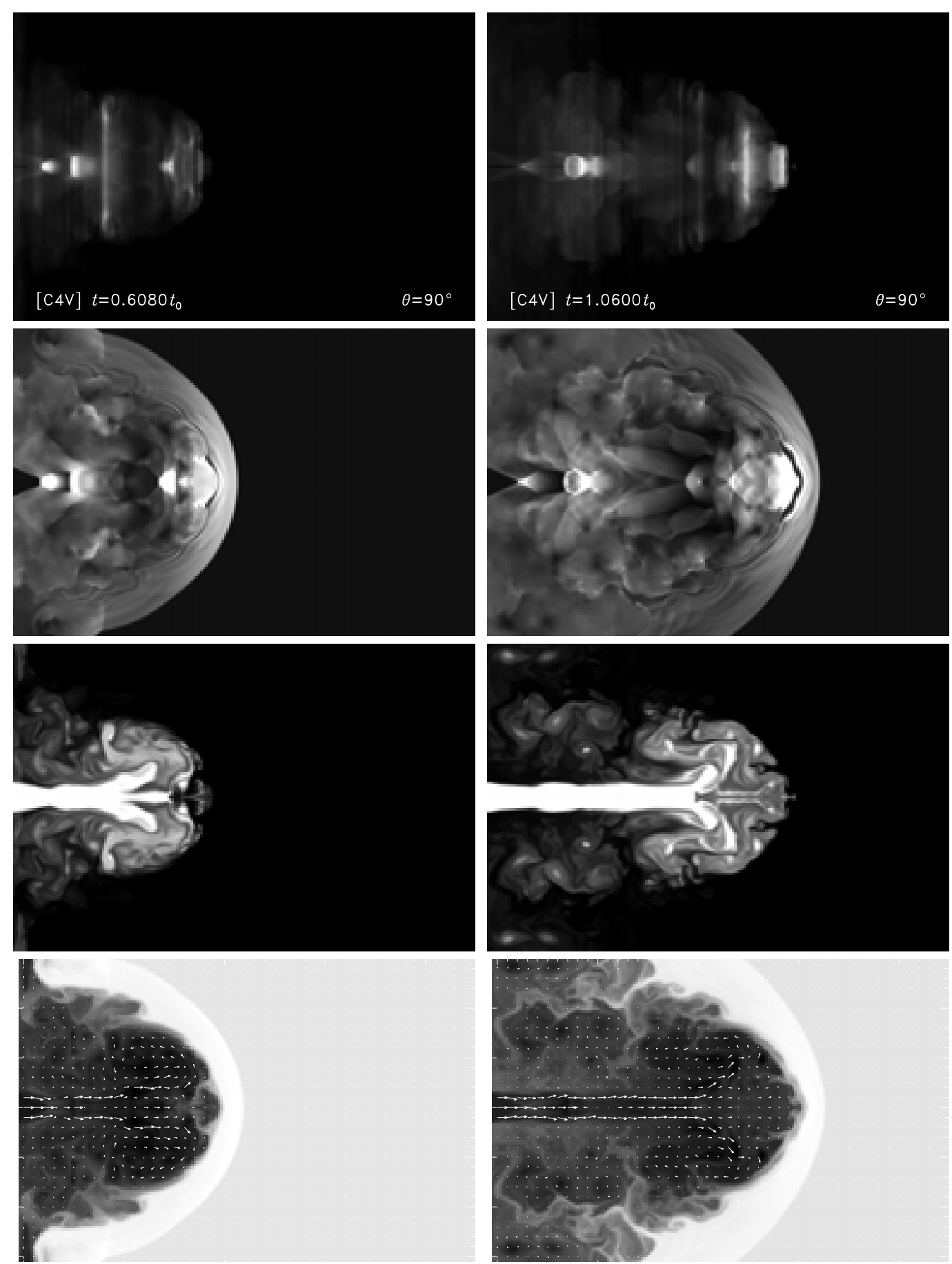

Fig. 21. Further examples of ray-traced frames resembling Pictor A, with jet parameters $(\eta, M)=\left(10^{-4}, 5\right)$. As in Fig. 20 , the rows from top to bottom show renderings, pressure, the tracer $\varphi$ and flow pattern.

In the second and third frames, the edge of the funnel has advanced outwards. In the third frame it collides with a flow of $\varphi \approx 0.5$ matter moving in the negative- $z$ direction near the outer surface of the cocoon. The flow of gas around the funnel causes a strong annular shock which also results in low presures at smaller $r$ and $z$. The shock produces significant emission, and the result is a bright bar visible in images rendered at $\theta \approx 90^{\circ}$.

During the activity that creates the bright ring, the diamond and transverse shocks moving in both directions within the jet produce several brighter knots of emission. The knots move and fluctuate in brightness, but they maintain an approximately constant separation. Frames 3 to 5 also show a hazy skirt of emission surrounding the jet to the left of the brightest knot; it is a backflowing, high- $\varphi$ body of fluid that previously came off the edge of the funnel.

In frames 5 and 6 the front hot-spot fades, as it spreads laterally, loses pressure and undergoes a reduction in $\varphi$ as a result of mixing. Meanwhile, the bright bar that persisted from frames 3 to 4 has also faded. The interaction between the funnel's edge and the well-mixed, outer-front backflow has 

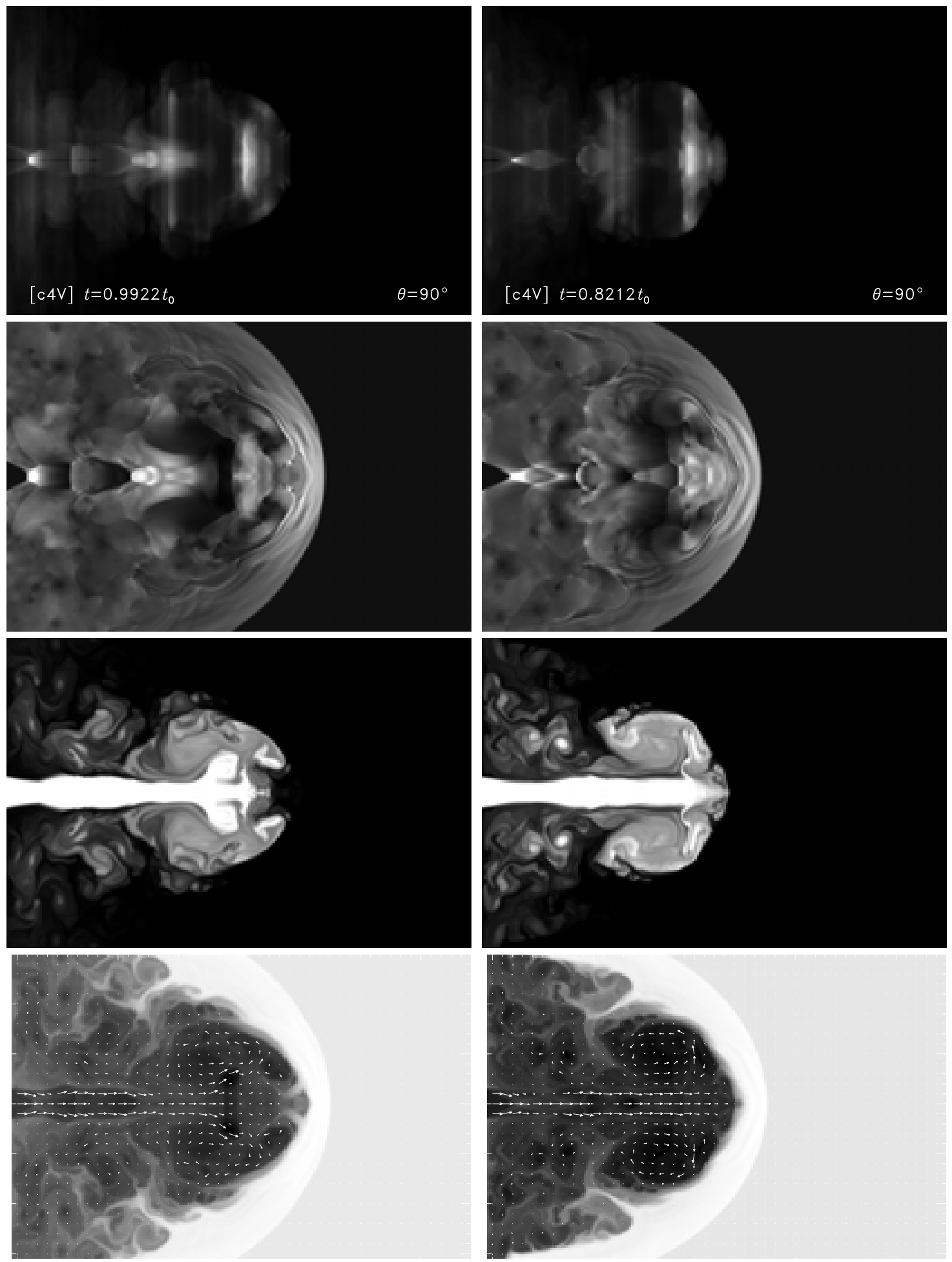

Fig. 22. Further examples of ray-traced frames resembling Pictor A, with jet parameters $(\eta, M)=\left(10^{-4}, 5\right)$. As in Fig. 20 , the rows from top to bottom show renderings, pressure, the tracer $\varphi$ and flow pattern.

progressed. The shocks of the initial interaction have separated and spread into a wider and complicated shock structure. The outer edge of the high- $\varphi$ funnel is swept in the negative- $z$, radially outwards direction by the well-mixed backflow from the head of the cocoon. In later frames (not shown), the funnel structure is greatly disrupted.

\section{Discussion}

\subsection{Orientation}

In this paper, we have presented a detailed analysis of simulations of light jets with a wide choice of parameters with the 

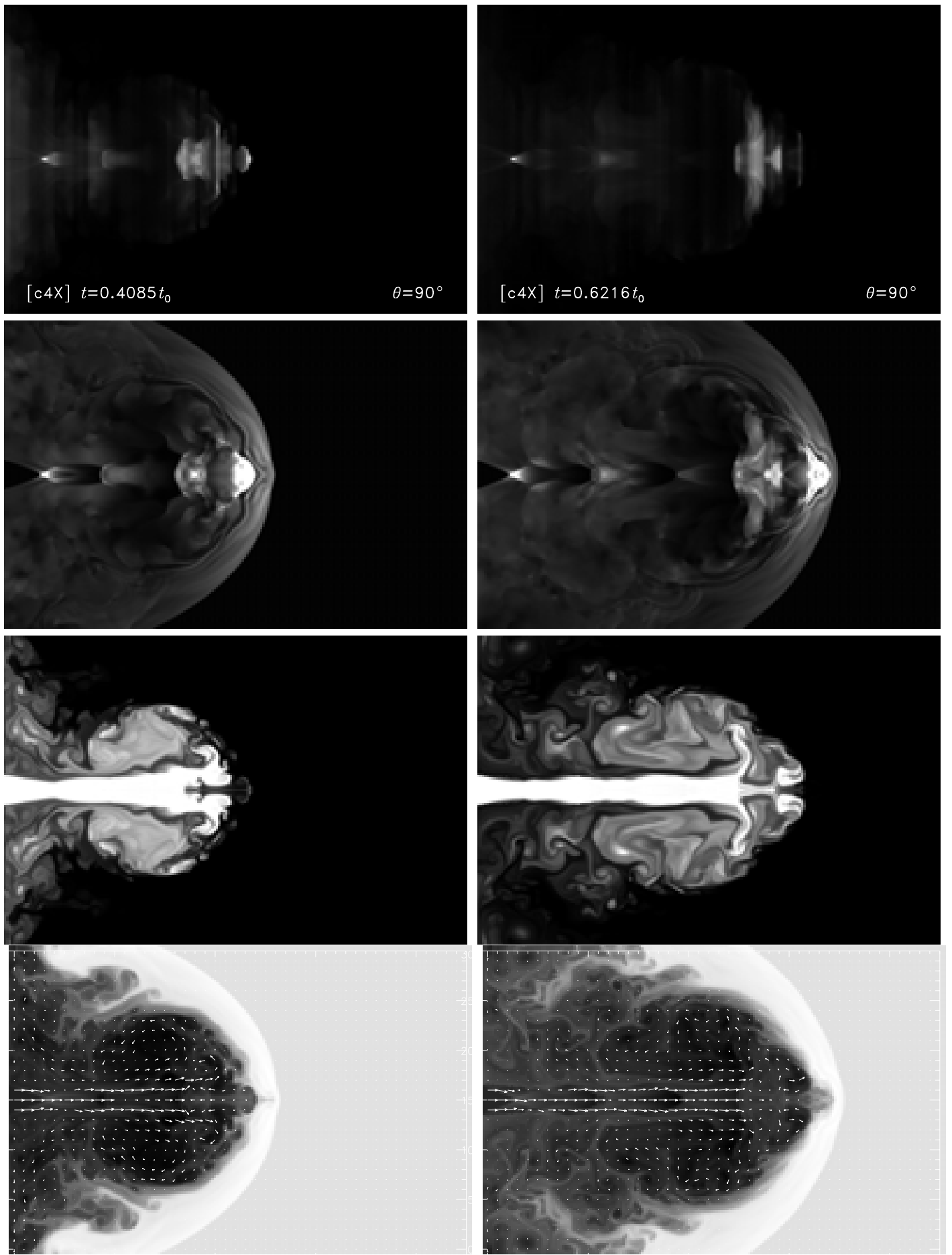

Fig. 23. Further examples of ray-traced frames resembling Pictor A, with jet parameters $(\eta, M)=\left(10^{-4}, 10\right)$. As in Fig. 20 , the rows from top to bottom show renderings, pressure, the tracer $\varphi$ and flow pattern.

main aim of determining the cause of the filament upstream of the western hot-spot in Pictor A. The dominant physical principle underlying this analysis is that complex terminal shock structure is manifest in simulations of light supersonic jets and that, in projection, such a shock complex may resemble the Pictor A radio and optical observations of the region near the western hot-spot. Similar complexity has been realised in three dimensional simulations of light supersonic jets by Tregillis et al. (2001). One of the advantges of restriction to a two dimensional simulation, at this stage, is the ability to map out a larger region of parameter space and to utilise higher spatial resolution. In particular, we have carried out simulations with a density ratio of $10^{-4}$; these would be quite expensive in three dimensions. 

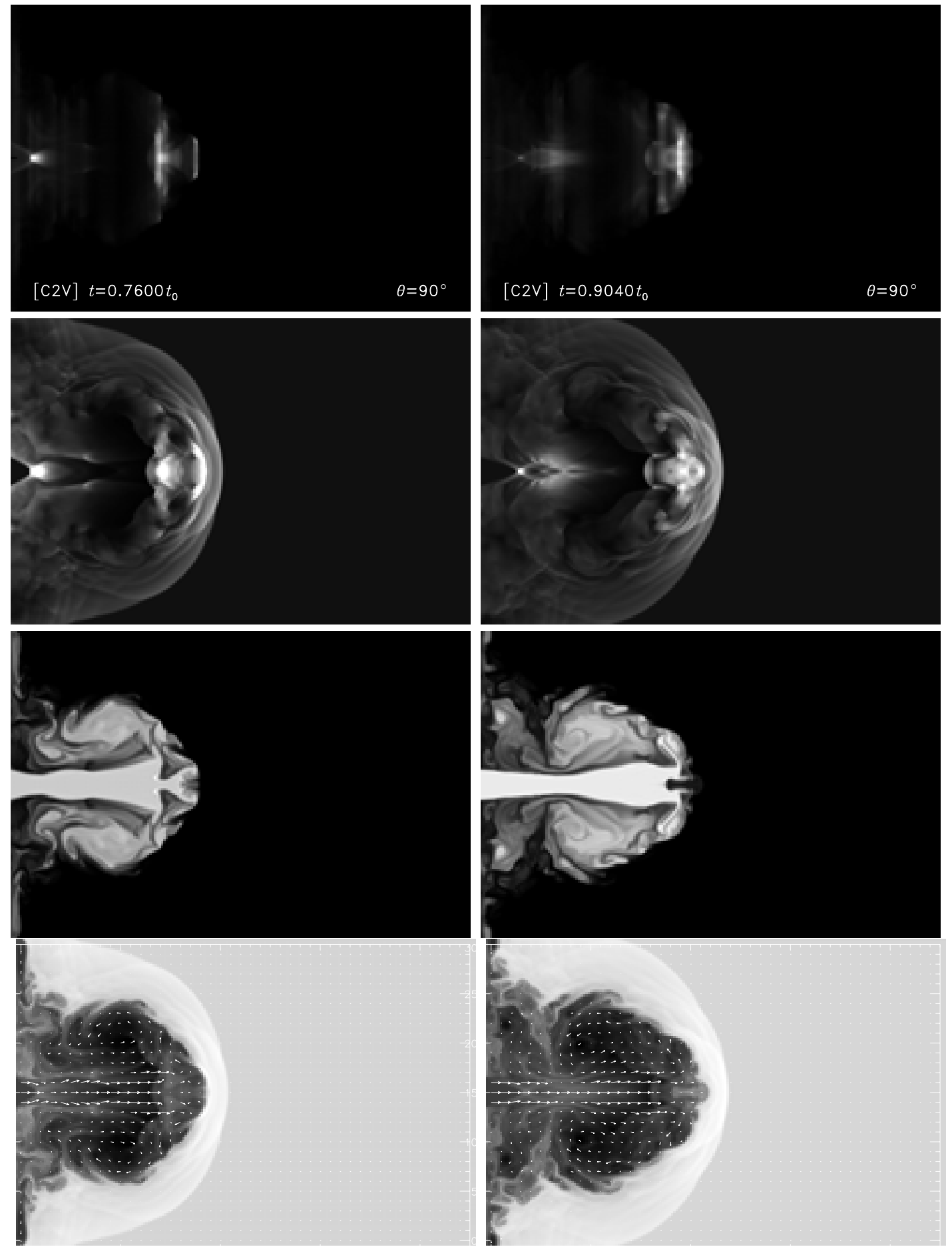

Fig. 24. $450 \times 300$ pixel sub-region of a jet with parameters $(\eta, M)=\left(10^{-2}, 5\right)$. As in Fig. 20 , the rows from top to bottom show renderings, pressure, the tracer $\varphi$ and flow pattern.

One of the most important constraints on the simulations is that the bar is a thin structure and if it is three dimensional, it would appear to be almost edge-on. Our rendering of synthetic radio images at different orientations bears this out. Adequate morphologies are not reproduced at low inclinations. Another decisive requirement that implies an almost edge-on structure, is reproduction of a filament with low curvature.
At inclinations $\theta<70^{\circ}$, geometric effects cannot convincingly produce a long, straight bar frequently enough. The projection of an annular structure with a ribbon-like cross-section makes one side brighter than the other, but the image is still significantly curved. The accidental coincidence of the near and far sides of separate rings in projection may give the appearance of a straight feature, but these tend to be narrower than 

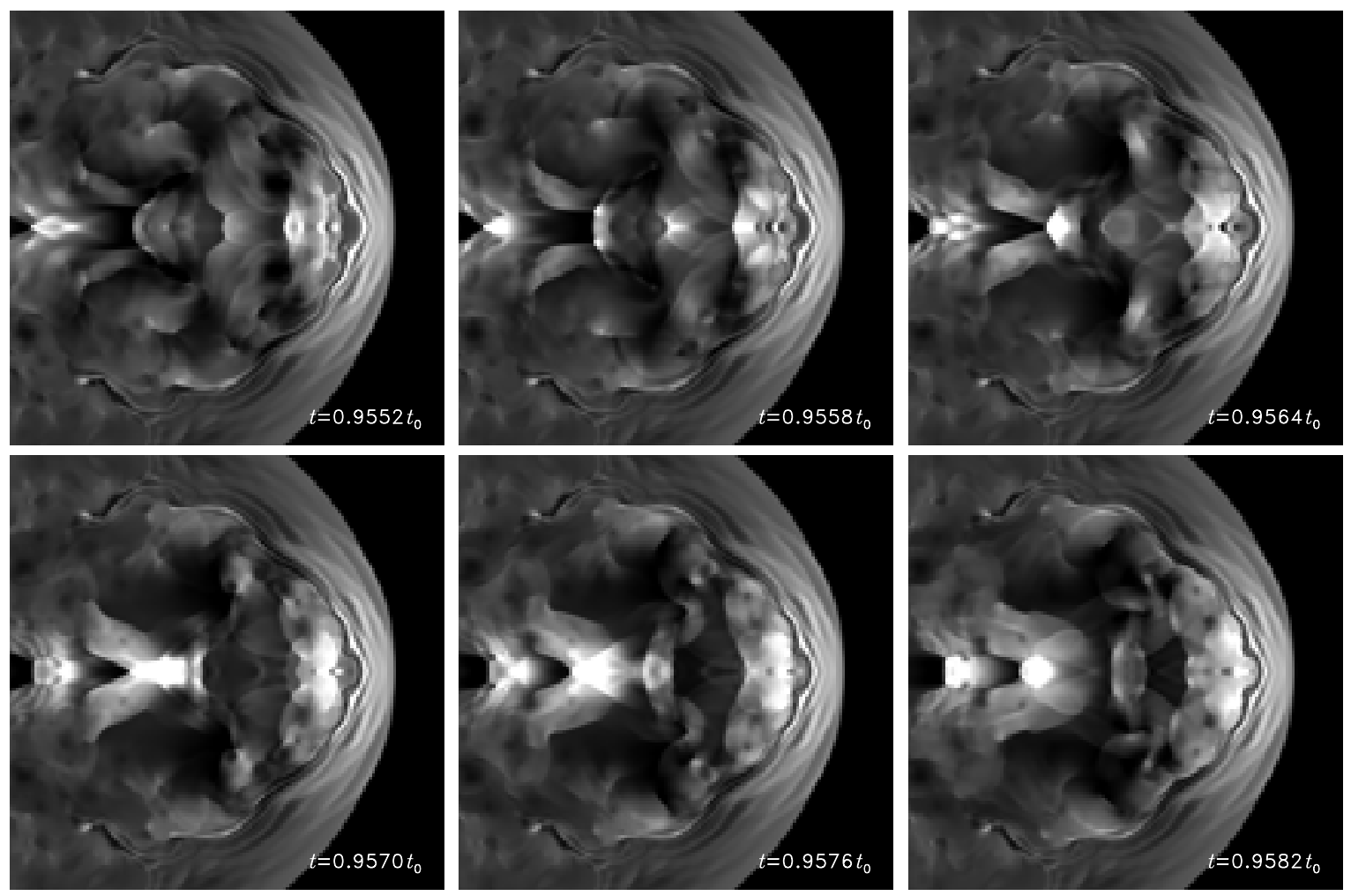

Fig. 25. Sequence of pressure snapshots during the emergence and disappearance of a particular configuration of bar and hot-spot. Jet parameters are $\left(10^{-4}, 5\right)$ and the left boundary is closed.

the observed filament. A structure with a very large radius of curvature might appear straight, but luminous structures of this type occur in our simulations in regions where the coocon is no more than $\sim 2 r_{\mathrm{j}}$ in radius. Three dimensional hydrodynamical effects may admit more complicated explanations for bar-like morphology. However extreme departures from axial symmetry in the backflow seem unlikely, given the strightness of the jet as indicated by the X-ray observations. On the basis of nonrelativistic hydrodynamics, we therefore think it likely that the inclination angle, $\theta>60^{\circ}$.

If there is any easy way to produce the appearance of a bar in a basically axisymmetric backflow viewed at $\theta<70^{\circ}$, it probably requires relativistic effects. A ring or disk moving relativistically appears more edge-on than if it were at rest (see Bicknell \& Begelman 1996). This would require the filament to be transient structure moving with a pattern speed larger than the expected hot-spot speed $\sim 0.1-0.2 c$. Relativistic beaming could also affect the brightness distribution if the matter passing through the filament and hot-spot shocks moves relativistically, brightening an annular structure on whichever side has a post-shock flow directed towards our line of sight. However the significance of these relativistic effects, for hot-spots in general and for Pictor A in particular, remains to be demonstrated, and is beyond the scope of our present study.

\subsection{Implications for physical parameters}

Although all choices of the jet parameters, jet density ratio, $\eta$, and Mach number, $M$, yield instants with appropriate morphology, the brightness characteristics of the resulting images may distinguish the most plausible values of the Mach number. Jets with larger Mach number typically produce terminal shock features with higher peak intensity. Perley et al. (1997) had deduced a high Mach number $(M \approx 40)$ for the jet in Pictor A based on the large ratio of the minimum pressures in the hot-spot and lobes. We have confirmed this in our simulations that show, for example, a brightness contrast $\sim 100$ for $(\eta, M)=\left(10^{-2}, 50\right)$ (see Sect. 5.2). Nevertheless, the unusual $(\eta, M)=\left(10^{-4}, 50\right)$ simulation produces a lower contrast and widely distributed rings in the backflow, and these features are clearly ruled out by observation. This may mean that the real jet parameters are less extreme, or else the powerfully turbulent backflow that occurs in this case may be less disruptive in three dimensions.

We do not find such a clear diagnostic of the density contrast of the jet. Cases with lower $\eta$ tend to produce broader cocoons, especially the rounded cavity in $\eta=10^{-4}$ simulations. For jets with larger $\eta$, the jet plasma and shocked thermal gas mix more rapidly in the cocoon. However all of the parameter sets we investigated permit the appearance of ephemeral barlike sructures with a range of widths, including some with $\approx 4 r_{\mathrm{j}}$ radius as required by the observations.

Many of our simulations imply relativistic bulk flow, $\beta \sim 1$ (see Table 2). One aspect of the observations where relativistic effects may be relevant concerns the large contrast between the hot-spot and radio lobe empshasised by Perley et al. (1997). On the basis of hydrodyamics alone, this would indicate a Mach number of approximately 40-50, or equivalently a Lorentz 

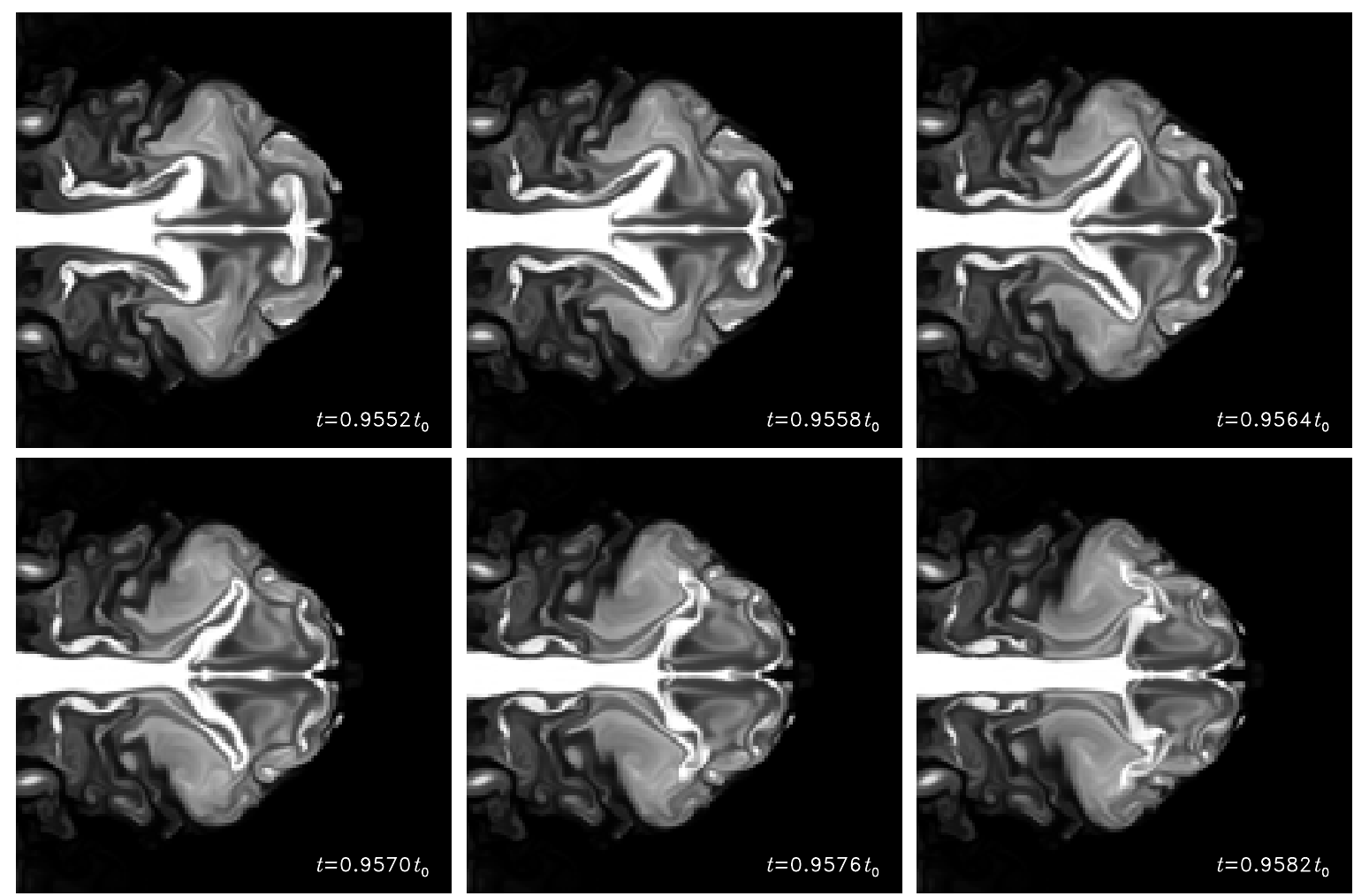

Fig. 26. Sequence of scalar tracer images, showing local relative concentration of jet plasma, corresponding to the snapshots in Fig. 25.

factor of approximately 25. This has motivated the $M=50$ simulations described above. Nevertheless, relativistic beaming may substantially contribute to the observed hot-spot brightness. This aspect may need to be taken into account with future simulations with a fully relativistic code.

\subsection{The orientation of the jet and filament in relation to radio and $X$-ray observations}

In their analysis of the Chandra X-ray Observatory data on Pictor A, Wilson et al. (2001) have suggested that an inclination $\theta \approx 23^{\circ}$ is viable. Larger angles are possible if jet magentic fields considerably less than equipartition are allowed. An inclination angle as small as $23^{\circ}$ would be in direct contradiction to our conclusion that the Pictor A radio structure is viewed at an angle of $\theta \gtrsim 70^{\circ}-90^{\circ}$. It is therefore worthwhile to examine the question of orientation in some detail.

The first part of the Wilson et al. (2001) argument relates to the arm-length ratio of the radio source. Assuming a velocity of advance for the hot-spot, $\beta_{\mathrm{HS}} \approx 0.11$ (Arshakian $\&$ Longair 2000) and the measured arm-length ratio of the source, Wilson et al. (2001) derive $\theta \approx 23^{\circ}$ but caution that this estimate by itself should not be taken too seriously for an individual source. The main limitation is probably the following: The statistical result for hot-spot advance includes sources in which the momentum of the jet is spread over a wide area as a result of the "Dentist Drill" effect (Scheuer 1982), thereby slowing its average advance. However, as we have remarked in justifying an axisymmetric simulation, the western jet in Pictor A appears to be very straight. That is, it does not appear to be substantially deflected and therefore the advance of the hotspot may be faster than the average Arshakian \& Longair (2000) value. Moreover, in contrast to the western lobe, the eastern lobe contains two hot-spots indicating some spreading of the jet force over a larger area than each hot-spot and a consequent slower expansion rate and larger arm-length ratio.

Now consider the effect of orientation on the X-ray emission and jet brightness ratio. As Wilson et al. (2001) remark, the X-ray emission resulting from scattering of the cosmic microwave background radiation by the electrons in the jet is proportional to $\delta^{4+2 \alpha}$ where $\delta$ is the Doppler factor and $\alpha \approx 0.9 \pm 0.5$ is the spectral index (Begelman \& Sikora 1987; Dermer 1995). The ratio of jet to counterjet X-ray brightnesses, $R \geq 10$ implies that $\beta \cos \theta \gtrsim 0.2$ for $\alpha=0.9$. The radio estimate of $R \gtrsim 3$ does not provide as stringent a limit $\beta \cos \theta \gtrsim 0.14$.

The main constraint on the angle $\theta$ is that it should not be so close to $90^{\circ}$ that the western jet is Doppler dimmed, in absolute terms, irrespective of the ratio of jet to counterjet fluxes. That is, $\delta$ should not be less than approximately 0.7 for an order of magnitude decrease in the X-ray flux.

For a given ratio, $R$, of jet to counterjet X-ray fluxes, we have

$\beta \cos \theta \approx \frac{R^{1 / p}-1}{R^{1 / p}+1}$

where $p=4+2 \alpha$ for inverse Compton emission off the microwave background. In Fig. 29, we show plots of the Doppler factor against inclination for values of $R=10,20$ and 50. An inclination of $70^{\circ}$ is consistent with $\delta \gtrsim 0.7$ for $R=10,20$ but not for $R=50$. If $\theta=70^{\circ}$ then Wilson et al. (2001) have shown 

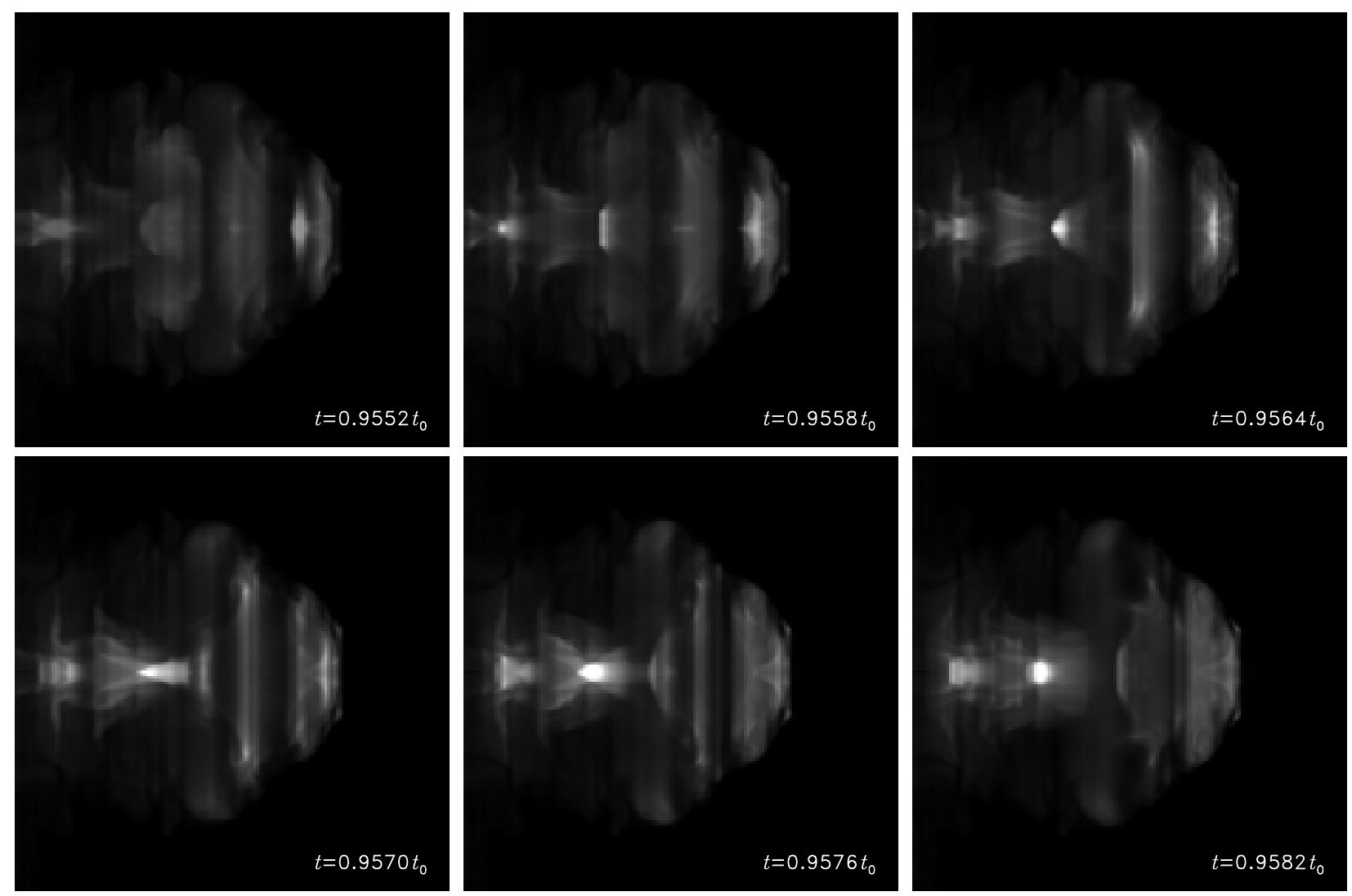

Fig. 27. Sequence of surface brightness images rendered at $\theta=90^{\circ}$, corresponding to the snapshots in Fig. 25.

that the magnetic field is about 0.03 times the minimum energy value. This constitutes no difficulty in principle since one does not necessarily expect minimum energy conditions to apply in the jet even though turbulent processes may be sufficient to produce minimum energy conditions in the lobes. Moreover, one possible estimate of the magnetic field in the hot-spot is an order of magnitude below equipartition. For reference, a jet inclination of $\theta=70^{\circ}$ and $R=10(20)$ implies a jet velocity of $\beta \approx 0.57(0.74)$. These estimates of velocity are consistent with other estimates in powerful sources (e.g. Wardle \& Aaron 1997). We conclude therefore that an orientation of $\theta \approx 70^{\circ}$ is consistent with the X-ray and radio constraints. We note, however, that the steep decline in the Doppler factor at around $\theta=70^{\circ}-80^{\circ}$ indicates that the constraints implied by the X-ray data are only just satisfied if $\theta=70^{\circ}$.

A jet velocity of $\beta \approx 0.6-0.7$ has other interesting ramifications since gas would then emerge from oblique shocks in the hot-spots with this velocity. Some of this gas would be moving directly towards us with $\delta \approx 3.1$ (for $\beta \approx 0.6$ ). The flux from that region of the hot-spot would be enhanced by a factor of 60 and this may be another explanation for the large ratio of hotspot surface brightness to lobe surface brightness (Perley et al. 1997).

If an almost side-on orientation proves to be untenable, then an alternative is that the limitations of an axisymmetric solution do not allow for a partial ring. That is, the ring could be brighter in one part than another as a result of three dimensional hydrodynamic effects. This may be consistent with the faint structures north of the filament seen in the greyscale image of the Perley et al. (1997) radio data, the Roeser (1989) optical image (see Fig. 3 of Wilson et al. 2001) and the VLT optical image (Fig. 2). These features may indicate a non-uniformly bright ring viewed at a more acute angle. We do not favour this explanation because of the straightness of the observed filament.

Another point in favour of $\theta \sim 90^{\circ}$ is that the western hotspot appears, in projection, at the edge of a wide lobe. If the source were to viewed at, say $\theta \approx 20^{\circ}$, then the hot-spot would most likely appear closer to the middle of the lobe. In addition such a small viewing angle makes the total extent of the source quite large $(\approx 1.3 \mathrm{Mpc})$. Radio galaxies this large are not unknown, but are fairly exceptional.

\subsection{Structure functions and hot-spot ratios}

The construction of cumulative distribution functions for hotspot ratios has been incidental to the main purpose of this paper. However, it is of general interest and quantifies the role of intrinsic variability in using such ratios for the estimation of say, relativistic effects.

The calculation of structure functions in jet simulations could have wide applicability. For example, the structure function of internal shocks could be applied to blazars.

Acknowledgements. This work was supported by an Australian Research Council Large Grant, A69905341 and grants of computing time from the ANU Supercomputer Facility. 

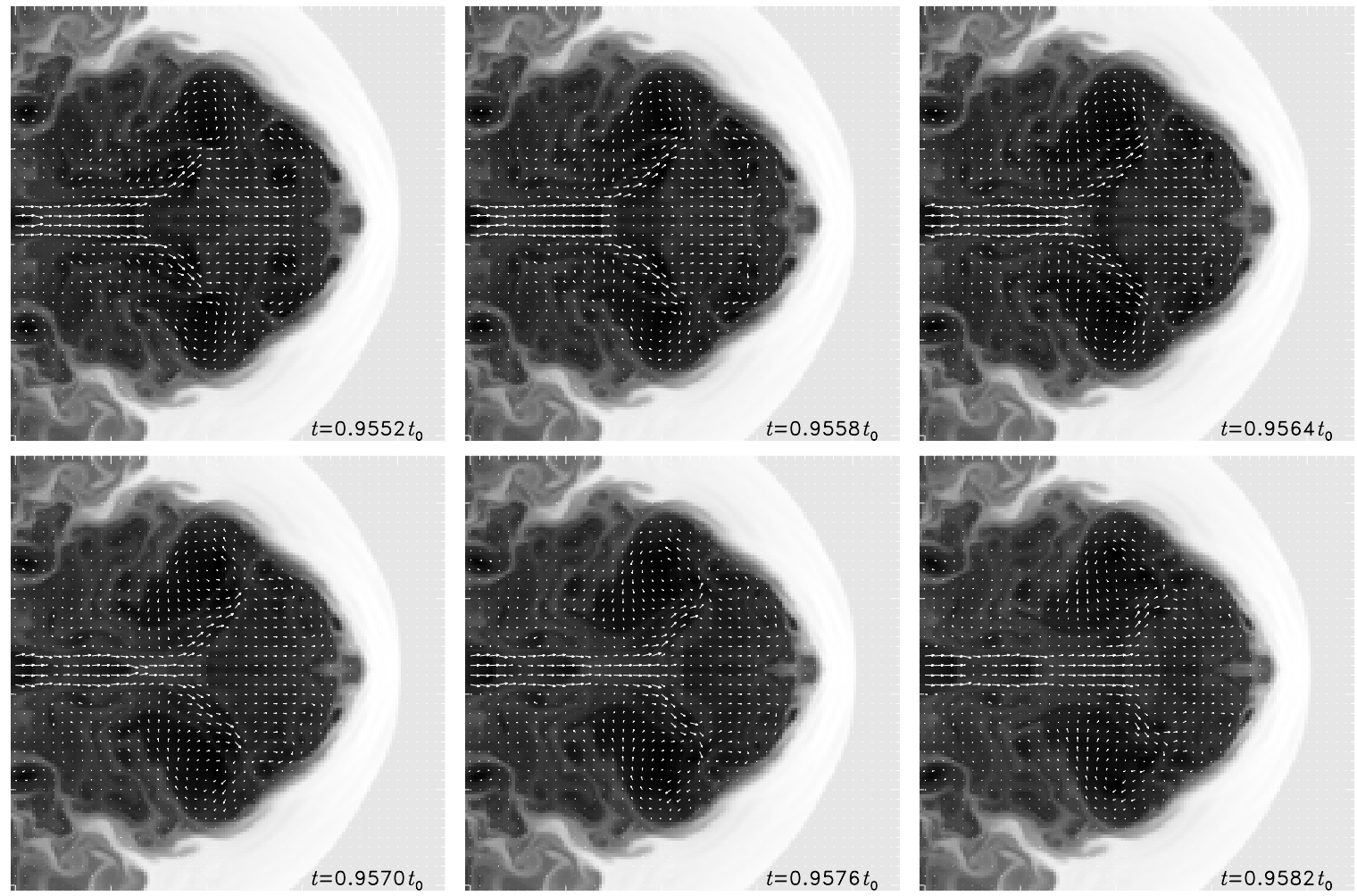

Fig. 28. Sequence of snapshots of the flow vectors superimposed on $\log \rho$ background, corresponding to the snapshots in Fig. 25 .

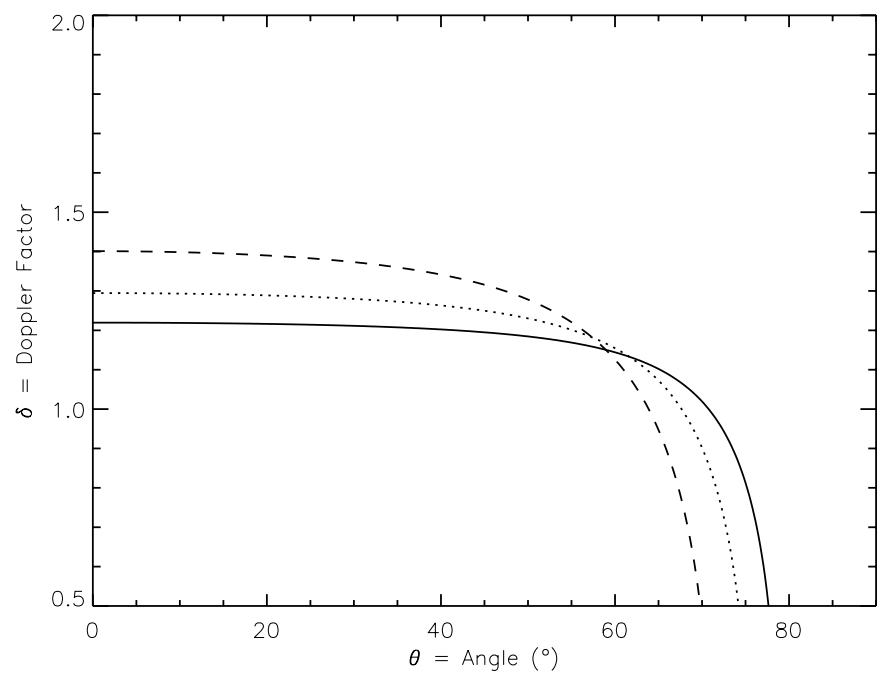

Fig. 29. The Doppler factor of the jet for a given ratio of jet to counterjet surface brightness. The cases $R=10,20,50$ are shown by solid, dotted and dashed lines respectively. Wilson et al. (2001) find $R>10$ for Pictor A.

\section{Appendix A: Probability distribution of hot-spot ratio}

In each simulation the bright features in the vicinity of the hotspot are unsteady, dynamic structures. The intrinsic variability of the peak intensity, $I$, of the hot-spot is easily quantified as a cumulative probability distribution function, $G(I)$. This function is numerically approximated by sorting the instantaneous $I$ values from a representative period $t_{0} \leq t \leq t_{0}+\Delta t$ of the simulation, in which $\Delta t \gg t_{\mathrm{dyn}}$. The sample must be representative in the sense that it begins and ends during the time in which the cocoon is well established and both the head of the jet and the bow shock are not close to any of the boundaries of the computational grid. If the intensity samples $\left\{I_{n}\right\}$ are ranked in ascending order, $\mathcal{R}_{n}$, for $n=1, \ldots, n_{\max }$, then the cumulative distribution function of the peak intensity is

$G(I) \equiv \operatorname{Pr}\left(I^{\prime} \leq I\right)=\frac{\mathcal{R}(I)}{n_{\max }}$

where $I$ is one of the sample values. If $I$ is not one of the sample values but has neighbouring values are, $I_{-}<I<I_{+}$, then $G(I)$ can be interpolated from $G\left(I_{-}\right)$and $G\left(I_{+}\right)$. Then we may define a probability density function,

$g(I)=\frac{\mathrm{d} G}{\mathrm{~d} I}$

which may be evaluated for any $I$ by numerical differentiation and/or interpolation as appropriate.

If the two jet and counter-jet have the same physical parameters and the respective hot-spots are separated by a distance exceeding $t_{\mathrm{dyn}} c$ then the hot-spots are causally independent. The probability distribution, $f(R)$, of the ratio, $R$, of hot-spot peak intensities can be derived from the intrinsic distributions of one hot-spot.

$$
\begin{aligned}
f(R) & \equiv \operatorname{Pr}\left(R I_{2}<I_{1}<(R+\mathrm{d} R) I_{2}\right) \\
& \equiv \frac{\mathrm{d} F}{\mathrm{~d} R}=\int_{0}^{\infty} g\left(R I_{2}\right) g\left(I_{2}\right) I_{2} \mathrm{~d} I_{2}
\end{aligned}
$$


Alternatively, the cumulative distribution function $F(R)$ may be calculated by taking a double sum over the sample of intensity values,

$F(R) \equiv \operatorname{Pr}\left(R^{\prime} \leq R\right)=\frac{1}{n_{\max ^{2}}} \sum_{i, j} H\left(R I_{j}-I_{i}\right)$,

where $H$ is the Heavyside step function $(H(x)=1$ for $x \geq 1$ and $H(x)=0$ otherwise).

\section{References}

Arshakian, T. G., \& Longair, M. S. 2000, MNRAS, 311, 846

Begelman, M. C., \& Sikora, M. 1987, ApJ, 322, 650

Bicknell, G. V., \& Begelman, M. C. 1996, ApJ, 467, 597

Birkhoff, G., \& Zarantonello, E. H. 1957, Jets, Wakes and Cavities (N.Y.: Academic Press)

Blandford, R. D., \& Rees, M. J. 1974, MNRAS, 169, 395

Blondin, J. M., \& Lukfin, E. A. 1993, ApJS, 88, 589

Carvalho, J. C., \& O’Dea, C. P. 2002, ApJS, 141, 337

Colella, P., \& Woodward, P. R. 1984, J. Comput. Phys., 54, 174
Dermer, C. D. 1995, ApJL, 446, L63

Norman, M. L., Smarr, L., Winkler, K.-H. A., \& Smith, M. D. 1982, A\&A, 113, 285

Perley, R. A., Röser, H. J., \& Meisenheimer, K. 1997, A\&A, 328, 12

Roeser, H. 1989, in Hot Spots in Extragalactic Radio Sources, 91

Rosen, A., Hughes, P. A., Duncan, G. C., \& Hardee, P. E. 1999, ApJ, 516, 729

Röser, H.-J., \& Meisenheimer, K. 1987, ApJ, 314, 70

Saxton, C. J., Sutherland, R. S., \& Bicknell, G. V. 2001, ApJ, 563, 103

Scheuer, P. A. G. 1982, in Extragalactic Radio Sources, ed. D. S. Heeschen, \& C. M. Wade (Dordrecht: Reidel), IAU Symp., 97, 163

Simonetti, J. H., Cordes, J. M., \& Heeschen, D. S. 1985, ApJ, 296, 46

Smith, M. D., Norman, M. L., Winkler, K.-H. A., \& Smarr, L. 1985, MNRAS, 214, 67

Tregillis, I. L., Jones, T. W., \& Ryu, D. 2001, ApJ, 557, 475

Wagner, S. J., Appenzeller, I., Bicknell, G. V., et al. 2002, in preparation

Wardle, J. F. C., \& Aaron, S. E. 1997, MNRAS, 286, 425

Wilson, A. S., Young, A. J., \& Shopbell, P. L. 2001, ApJ, 547, 740 